\title{
Use of power transformation for estimating the population mean in presence of non-response in successive sampling
}

\author{
S. K. PAL AND H. P. SINGH
}

\begin{abstract}
This paper addresses the problem of estimating the population mean at the current occasion in two occasion successive sampling when non-response occurs on the current (second) occasions. Using the power transformation we have suggested classes of estimators of current population mean and their properties are studied. Optimum replacement strategies for the proposed estimators have been given and empirical studies are carried out to assess the performance of estimators. We have made suitable recommendation to the practitioners on the basis of the empirical study.
\end{abstract}

Mathematics Subject Classification 2000: $62 \mathrm{D05}$

Keywords: Auxiliary variate, Study variate, Bias, Mean squared error, Non- response, Efficiency.

\section{INTRODUCTION}

It is fact that the use of auxiliary information in the study of sample survey gives an efficient estimate of population parameter like population mean or total, under some conditions. The estimation of the population mean is an important issue in sampling theory and several efforts have been made to improve the precision of the estimates. The most common method of data collection in survey research is sending the questionnaire through mail. The reason may be the minimum cost involved in this method. But this method has a major disadvantage that, a large rate of non-response may occur which may result in an unknown bias at any assumption because of the fact that the estimate based only on responding units is representative of the both responding and non-responding units. Personal interview is another method of data collection, which generally may result in a complete response, but the cost involved in personal interviews is much higher than the mail questionnaire method. We may conclude from the above discussion that the advantage of one method is the disadvantage of the other vice-versa. Hansen and Hurwitz (1946) 
combined the advantage of both procedures. They considered a problem to determine the number of mail questionnaires along with the number of personal interviews to take in following up non-response to the mail questionnaire in order to attain the required precision at minimum cost.

The method of collecting information on selected part of population is termed as sampling survey. There are various useful examples, where the survey often needs to be repeated many times. The main purpose of repeated survey is to allow one or more items to be monitored over time.

For the intention of survey design, the aim has often been simplified to two objectives: to produce the reliable estimates of the item on each occasion, and to generate practical estimates of change from occasion to occasion. Sampling on successive occasions with a partial replacement of sampling units was first considered by Jessen (1942) in the analysis of farm data. He pioneered using the whole information gathered in the previous occasions. The theory of successive sampling was further developed by Patterson (1950), Rao and Graham (1964), Singh et al. (1992), Feng and Zou (1997), Biradar and Singh (2001), Singh and Vishwakarma (2007a, b, 2009), Singh and Pal (2015a, b, c, d) and Singh and Pal (2016a, b, c) among others. Generally almost all surveys suffer from the problem of non-response. Lack of information, absence at the time of survey, and refusal of the respondents are main reason of the non-response. However, an extensive description of the different types of non-response and their effects on surveys could be found in Cochran (1977). Hansen and Hurwitz (1946) considered the problem of nonresponse while estimating the population mean by taking a subsample from the nonrespondents group with the help of some extra efforts and an estimator was suggested by combining the information available from response and non-response groups. Recently Chaudhary et al. (2004), Singh and Priyanka (2007) Singh and Kumar (2008, 2010 and 2011) and Singh et al.(2011) used the Hansen and Hurwitz (1946) technique for the estimation of population mean on current occasion in two occasion successive sampling in the presence of non-response.

The aim of the present work is to study the effect of non-response, when it occurs on current occasion in two occasion successive (rotation) sampling. 


\section{THE TECHNIQUE}

Let $U=\left(U_{1}, U_{2}, \ldots, U_{N}\right)$ be a finite population of $\mathrm{N}$ units, which has been sampled over two occasions. The character under study is denoted by $x(y)$ on the first (second) occasion. It is assumed that information on an auxiliary variable $z$ (with unknown population mean), which is positively correlated with the study variable, is readily available and almost stable over both the occasions. A simple random sample (without replacement) $s_{n}$ of $\mathrm{n}$ units is drawn on the first occasion. A random sub-sample $s_{m}$ of $m=n \lambda$ units is retained (matched) for its use on the second occasion. We assume that there is non-response at the current occasion, so that the population can be divided into two classes, those who will respond at the first attempt and those who will not respond. Let the sizes of these two classes be $N_{1}$ and $N_{2}$, respectively. At the current (second) occasion a simple random sample (without replacement) $\underline{s}_{u}$ of $u=(n-m)=n \mu$ units is drawn afresh from the entire population so that the sample size on the current (second) occasion is also $n$. $\lambda$ and $\mu$ $(\lambda+\mu=1)$ are the fractions of matched and fresh samples, respectively, at the current (second) occasion $u_{1}$ units respond and $u_{2}$ units do not respond. Let $u_{2 h}$ denote the size of the subsample $S_{u 2 h}$ drawn from the non-responding units in the unmatched (fresh) portion of the sample $\left(s_{u}\right)$ on the current (second) occasion. The following notations are used:

$\bar{X}, \bar{Y}, \bar{Z}:$ The population means of the variable $x, y$ and $z$ respectively, $\bar{y}_{m}, \bar{y}_{u}, \bar{y}_{u_{1}}, \bar{y}_{u_{2 h}}, \bar{y}_{n}, \bar{y}_{n_{1}}, \bar{y}_{n_{2 h}}, \bar{x}_{n}, \bar{x}_{m}, \bar{z}_{m}, \bar{z}_{u}, \bar{z}_{u_{1}}, \bar{z}_{u_{2 h}}:$ The sample means of the respective variables based on the sample sizes shown in subscripts.

$\rho_{y x}, \rho_{x z}, \rho_{y z}:$ The population correlation coefficient between the variables shown in the subscripts.

$\rho_{y x(2)}$ : The population correlation coefficient between $y$ and $x$ in the nonresponding units of the population.

$\rho_{y z(2)}$ : The population correlation coefficient between $y$ and $z$ in the non-responding units of the population. 
$\rho_{x z(2)}$ : The population correlation coefficient between $x$ and $z$ in the non-responding units of the population.

$S_{x}^{2}, S_{y}^{2}, S_{z}^{2}$ : The population variances of the variable $x, y$ and $z$ respectively.

$S_{y(2)}^{2}, S_{z(2)}^{2}$ : The population variances of the variable $y$ and $z$ respectively, in the non-responding units of the population.

$W\left(=N_{2} / N\right)$ : The proportion of non-responding units in the population at current (second) occasion.

$\bar{y}_{u}^{*}=\frac{u_{1} \bar{y}_{u 1}+u_{2} \bar{y}_{u 2 h}}{u}$ and $\bar{z}_{u}^{*}=\frac{u_{1} \bar{z}_{u 1}+u_{2} \bar{z}_{u 2 h}}{u}:$ Hansen and Hurwitz estimators of study variable $y$ and auxiliary variable $x$, respectively for the unmatched portion of the sample on the current occasions. $f_{2}\left(=u_{2} / u_{2 h}\right)$ and $f_{2}^{*}\left(=n_{2} / n_{2 h}\right)$.

\subsection{Formulation of Estimator}

To estimate the population mean $\bar{Y}$ on the current (second) occasion, two different sets of estimates are considered that use information on a stable auxiliary variable $z$. Single set of estimators $S_{u}=\left(P_{1 u}, P_{2 u}\right)$ based on sample $s_{u}$ of size $u$ drawn afresh on the second occasion and the second set of estimates $S_{m}=P_{m}$ based on the sample $s_{m}$ of size $m$ which is common to both the occasions. Since the nonresponse occurs in the sample $s_{u}$, therefore, we have used the Hansen and Hurwitz (1946) technique to propose the estimators of sets $s_{u}$. For this reason the estimators of sets $s_{u}$ and $s_{m}$ for estimating the current population mean $\bar{Y}$ are formulated as

$$
\begin{aligned}
& P_{1 u}=\bar{y}_{u}^{*}\left(\frac{\bar{Z}}{\bar{z}_{u}}\right)^{\eta} \\
& P_{2 u}=\bar{y}_{u}^{*}\left(\frac{\bar{Z}}{\bar{z}_{u}^{*}}\right)^{\eta}
\end{aligned}
$$

and

$$
P_{m}=\bar{y}_{m}\left(\frac{\bar{x}_{n}}{\bar{x}_{m}}\right)^{\eta}\left(\frac{\bar{Z}}{\bar{z}_{m}}\right)^{\eta}
$$


where $\eta$ is a scalar such that $0 \leq \eta \leq 1$.

Combining the estimators of sets $S_{u}$ and $S_{m}$, we have the following estimators of the population mean $\bar{Y}$ at the current (second) occasion.

$$
P_{i}=\varphi_{i} P_{i u}+\left(1-\varphi_{i}\right) P_{m} ;(i=1,2)
$$

where $\varphi_{\mathrm{i}}$ 's $\left(0 \leq \varphi_{i} \leq 1\right)(i=1,2)$ are unknown constants (scalars) to be determined such that MSE of $P_{i}$ 's $(i=1,2)$ are least.

\subsection{Properties of The Estimator}

The bias and mean squared errors (MSEs) of the estimators $P_{i}(i=1,2)$ are obtained up to the first degree of approximation using the following adaptation:

$$
\begin{aligned}
& \bar{y}_{u}^{*}=\bar{Y}\left(1+e_{0 u}^{*}\right), \bar{y}_{m}=\bar{Y}\left(1+e_{0 m}\right), \bar{x}_{m}=\bar{X}\left(1+e_{1 m}\right), \\
& \bar{x}_{n}=\bar{X}\left(1+e_{1 n}\right), \bar{z}_{u}^{*}=\bar{Z}\left(1+e_{2 u}^{*}\right), \bar{z}_{u}=\bar{Z}\left(1+e_{2 u}\right) \text { and } \bar{z}_{m}=\bar{Z}\left(1+e_{2 m}\right) .
\end{aligned}
$$

such that

$$
E\left(e_{0 u}^{*}\right)=E\left(e_{0 m}\right)=E\left(e_{1 m}\right)=E\left(e_{1 n}\right)=E\left(e_{2 u}^{*}\right)=E\left(e_{2 u}\right)=E\left(e_{2 m}\right)=0
$$

and

$$
\begin{aligned}
& E\left(e_{0 u}^{* 2}\right)=\left[\left(\frac{1}{u}-\frac{1}{N}\right) C_{y}^{2}+\frac{W\left(f_{2}-1\right)}{u} C_{y(2)}^{2}\right], E\left(e_{0 m}^{2}\right)=\left(\frac{1}{m}-\frac{1}{N}\right) C_{y}^{2}, \\
& E\left(e_{1 m}^{2}\right)=\left(\frac{1}{m}-\frac{1}{N}\right) C_{x}^{2}, \\
& E\left(e_{1 n}^{2}\right)=\left(\frac{1}{n}-\frac{1}{N}\right) C_{x}^{2}, E\left(e_{2 u}^{* 2}\right)=\left[\left(\frac{1}{u}-\frac{1}{N}\right) C_{z}^{2}+\frac{W\left(f_{2}-1\right)}{u} C_{z(2)}^{2}\right], \\
& E\left(e_{2 u}^{2}\right)=\left(\frac{1}{u}-\frac{1}{N}\right) C_{z}^{2}, E\left(e_{2 m}^{2}\right)=\left(\frac{1}{m}-\frac{1}{N}\right) C_{z}^{2} ; E\left(e_{0 u}^{*}, e_{0 m}\right)=-\frac{1}{N} C_{y}^{2}, \\
& E\left(e_{0 u}^{*}, e_{1 m}\right)=-\frac{1}{N} \rho_{y x} C_{y} C_{x}, E\left(e_{0 u}^{*}, e_{1 n}\right)=-\frac{1}{N} \rho_{y x} C_{y} C_{x}, \\
& E\left(e_{0 u}^{*}, e_{2 u}^{*}\right)=\left[\left(\frac{1}{u}-\frac{1}{N}\right) \rho_{y z} C_{y} C_{z}+\frac{W\left(f_{2}-1\right)}{u} \rho_{y z(2)} C_{y(2)} C_{z(2)}\right], \\
& E\left(e_{0 u}^{*}, e_{2 u}\right)=\left(\frac{1}{u}-\frac{1}{N}\right) \rho_{y z} C_{y} C_{z}, E\left(e_{0 u}^{*}, e_{2 m}\right)=-\frac{1}{N} \rho_{y z} C_{y} C_{z} ;
\end{aligned}
$$




$$
\begin{aligned}
& E\left(e_{0 m}, e_{1 m}\right)=\left(\frac{1}{m}-\frac{1}{N}\right) \rho_{y x} C_{y} C_{x}, E\left(e_{0 m}, e_{1 n}\right)=\left(\frac{1}{n}-\frac{1}{N}\right) \rho_{y x} C_{y} C_{x}, \\
& E\left(e_{0 m}, e_{2 u}^{*}\right)=-\frac{1}{N} \rho_{y z} C_{y} C_{z}, E\left(e_{0 m}, e_{2 u}\right)=-\frac{1}{N} \rho_{y z} C_{y} C_{z}, \\
& E\left(e_{0 m}, e_{2 m}\right)=\left(\frac{1}{m}-\frac{1}{N}\right) \rho_{y z} C_{y} C_{z} ; E\left(e_{1 m}, e_{1 n}\right)=\left(\frac{1}{n}-\frac{1}{N}\right) C_{x}^{2}, \\
& E\left(e_{1 m}, e_{2 u}^{*}\right)=-\frac{1}{N} \rho_{x z} C_{x} C_{z}, E\left(e_{1 m}, e_{2 u}\right)=-\frac{1}{N} \rho_{x z} C_{x} C_{z}, \\
& E\left(e_{1 m}, e_{2 m}\right)=\left(\frac{1}{m}-\frac{1}{N}\right) \rho_{x z} C_{x} C_{z} ; E\left(e_{1 n}, e_{2 u}^{*}\right)=-\frac{1}{N} \rho_{x z} C_{x} C_{z}, \\
& E\left(e_{1 n}, e_{2 u}\right)=-\frac{1}{N} \rho_{x z} C_{x} C_{z}, E\left(e_{1 n}, e_{2 m}\right)=\left(\frac{1}{n}-\frac{1}{N}\right) \rho_{x z} C_{x} C_{z} ; \\
& E\left(e_{2 u}^{*}, e_{2 u}\right)=\left(\frac{1}{u}-\frac{1}{N}\right) C_{z}^{2}, E\left(e_{2 u}^{*}, e_{2 m}\right)=-\frac{1}{N} C_{z}^{2}, E\left(e_{2 u}, e_{2 m}\right)=-\frac{1}{N} C_{z}^{2} ;
\end{aligned}
$$

where

$$
\begin{aligned}
& C_{y}=\frac{S_{y}}{\bar{Y}}, C_{x}=\frac{S_{x}}{\bar{X}}, C_{z}=\frac{S_{z}}{\bar{Z}}, C_{y(2)}=\frac{S_{y(2)}}{\bar{Y}}, C_{x(2)}=\frac{S_{x(2)}}{\bar{X}}, C_{z(2)}=\frac{S_{z(2)}}{\bar{Z}}, \\
& \rho_{y x}=\frac{S_{y x}}{\left(S_{y} S_{x}\right)}, \rho_{y z}=\frac{S_{y z}}{\left(S_{y} S_{z}\right)}, \rho_{y z(2)}=\frac{S_{y z(2)}}{\left(S_{y(2)} S_{z(2)}\right)}, \rho_{y x(2)}=\frac{S_{y x(2)}}{\left(S_{y(2)} S_{x(2)}\right)}, \\
& S_{y}^{2}=\frac{1}{(N-1)} \sum_{i=1}^{N}\left(y_{i}-\bar{Y}\right)^{2}, S_{x}^{2}=\frac{1}{(N-1)} \sum_{i=1}^{N}\left(x_{i}-\bar{X}\right)^{2}, S_{z}^{2}=\frac{1}{(N-1)} \sum_{i=1}^{N}\left(z_{i}-\bar{Z}\right)^{2} \\
& S_{y x}=\frac{1}{(N-1)} \sum_{i=1}^{N}\left(y_{i}-\bar{Y}\right)\left(x_{i}-\bar{X}\right), S_{y z}=\frac{1}{(N-1)} \sum_{i=1}^{N}\left(y_{i}-\bar{Y}\right)\left(z_{i}-\bar{Z}\right), \\
& S_{y(2)}^{2}=\frac{1}{\left(N_{2}-1\right)} \sum_{i=1}^{N_{2}}\left(y_{i}-\bar{Y}_{(2)}\right)^{2}, \bar{Y}_{(2)}=\frac{1}{N_{2}} \sum_{i=1}^{N_{2}} y_{i} S_{z(2)}^{2}=\frac{1}{\left(N_{2}-1\right)} \sum_{i=1}^{N_{2}}\left(z_{i}-\bar{Z}_{(2)}\right)^{2}, \\
& S_{y z(2)}=\frac{1}{\left(N_{2}-1\right)} \sum_{i=1}^{N_{2}}\left(y_{i}-\bar{Y}_{(2)}\right)\left(z_{i}-\bar{Z}_{(2)}\right), \bar{Z}_{(2)}=\frac{1}{N_{2}} \sum_{i=1}^{N_{2}} z_{i} .
\end{aligned}
$$


Under the above adaptations estimators $P_{i u}(i=1,2)$ and $P_{m}$ approximately take the following forms

$$
\begin{aligned}
P_{1 u} & \cong \bar{Y}\left[1+e_{0 u}^{*}-\eta e_{2 u}-\eta e_{0 u}^{*} e_{2 u}+\frac{\eta(\eta+1)}{2} e_{2 u}^{2}\right] \\
P_{2 u} & \cong \bar{Y}\left[1+e_{0 u}^{*}-\eta e_{2 u}^{*}-\eta e_{0 u}^{*} e_{2 u}^{*}+\frac{\eta(\eta+1)}{2} e_{2 u}^{* 2}\right] \\
P_{m} & \cong \bar{Y}\left[1+e_{0 m}+\eta\left(e_{1 n}-e_{1 m}-e_{2 m}\right)+\eta\left(e_{0 m} e_{1 n}-e_{0 m} e_{2 m}-e_{0 m} e_{1 m}\right)\right. \\
& \left.+\eta^{2}\left(e_{1 m} e_{1 m}-e_{2 m} e_{1 n}-e_{1 m} e_{1 n}\right)+\frac{\eta(\eta-1)}{2} e_{1 n}^{2}+\frac{\eta(\eta+1)}{2} e_{1 m}^{2}+\frac{\eta(\eta+1)}{2} e_{2 m}^{2}\right]
\end{aligned}
$$

THEOREM 2.1: Bias of the estimator $P_{i}(i=1,2)$ to the first degree of approximation are obtained as

$$
B\left(P_{i}\right)=\varphi_{i} B\left(P_{i u}\right)+\left(1-\varphi_{i}\right) B\left(T_{m}\right),(i=1,2)
$$

where

$$
\begin{aligned}
& B\left(P_{1 u}\right)=\left(\frac{\eta \bar{Y}}{2}\right)\left(\frac{1}{u}-\frac{1}{N}\right) C_{z}^{2}\left(\eta-2 k_{y x}+1\right) \\
& B\left(P_{2 u}\right)=\left(\frac{\eta \bar{Y}}{2}\right)\left[\left(\frac{1}{u}-\frac{1}{N}\right) C_{z}^{2}\left(\eta-2 k_{y x}+1\right)+\frac{W\left(f_{2}-1\right)}{u} C_{z(2)}^{2}\left(\eta-2 k_{y x(2)}+1\right)\right] \\
& B\left(P_{m}\right)=\left(\frac{\eta \bar{Y}}{2}\right)\left[\left(\frac{1}{m}-\frac{1}{N}\right)\left\{C_{z}^{2}\left(\eta-2 k_{y x}+1\right)\right\}+\left(\frac{1}{m}-\frac{1}{n}\right) C_{x}^{2}\left\{1-2 k_{y x}+\eta\left(1+2 k_{z x}\right)\right\}\right]
\end{aligned}
$$

where $k_{y x}=\rho_{y x}\left(\frac{C_{y}}{C_{x}}\right), k_{y x(2)}=\rho_{y x(2)}\left(\frac{C_{y(2)}}{C_{x(2)}}\right), k_{y z}=\rho_{y z}\left(\frac{C_{y}}{C_{z}}\right)$,

$$
k_{z x}=\rho_{z x}\left(\frac{C_{z}}{C_{x}}\right), k_{x z}=\rho_{x z}\left(\frac{C_{x}}{C_{z}}\right) .
$$

PROOF: The bias of the estimator $P_{i}$ 's $(i=1,2)$ are given by

$$
\begin{aligned}
B\left(P_{i}\right)=E\left(P_{i}-\bar{Y}\right) & =\varphi_{i} E\left[P_{i u}-\bar{Y}\right]+\left(1-\varphi_{i}\right) E\left(P_{m}-\bar{Y}\right) \\
& =\varphi_{i} B\left(P_{i u}\right)+\left(1-\varphi_{i}\right) B\left(P_{m}\right)
\end{aligned}
$$

where $B\left(P_{i u}\right)=E\left(P_{i u}-\bar{Y}\right)$ and $B\left(P_{m}\right)=E\left(P_{m}-\bar{Y}\right)$. 
Substituting the expressions of $P_{1 u}, P_{2 u}$ and $P_{m}$ from (2.5), (2.6) and (2.7) in (2.8) taking expectations and retaining the terms up to the first order of sample sizes, we have the expressions for the bias of the estimators $P_{i}(i=1,2)$ as described in (2.9).

THEOREM 2.2: Mean squared error $(M S E)$ of the estimators $P_{i}(i=1,2)$ to the first degree of approximation are obtained as

$$
\operatorname{MSE}\left(P_{i}\right)=\varphi_{i}^{2} \operatorname{MSE}\left(P_{i u}\right)+\left(1-\varphi_{i}\right)^{2} \operatorname{MSE}\left(P_{m}\right)+2 \varphi_{i}\left(1-\varphi_{i}\right) \operatorname{Cov}\left(P_{i u}, P_{m}\right),(i=1,2)
$$

where

$$
\begin{aligned}
\operatorname{MSE}\left(P_{1 u}\right)= & \bar{Y}^{2}\left[\left(\frac{1}{u}-\frac{1}{N}\right)\left\{C_{y}^{2}+\eta C_{z}^{2}\left(\eta-2 k_{y z}\right)\right\}+\frac{W\left(f_{2}-1\right)}{u} C_{y(2)}^{2}\right] \\
\operatorname{MSE}\left(P_{2 u}\right)= & \bar{Y}^{2}\left[\left(\frac{1}{u}-\frac{1}{N}\right)\left\{C_{y}^{2}+\eta C_{z}^{2}\left(\eta-2 k_{y z}\right)\right\}\right. \\
& \left.+\frac{W\left(f_{2}-1\right)}{u}\left\{C_{y(2)}^{2}+\eta C_{z(2)}^{2}\left(\eta-2 k_{y z(2)}\right)\right\}\right] \\
\operatorname{MSE}\left(P_{m}\right)= & \bar{Y}^{2}\left[\left(\frac{1}{m}-\frac{1}{N}\right)\left\{C_{y}^{2}+\eta C_{z}^{2}\left(\eta-2 k_{y z}\right)\right\}\right. \\
& \left.+\left(\frac{1}{m}-\frac{1}{n}\right) \eta C_{x}^{2}\left\{\eta\left(1+2 k_{x z}\right)-2 k_{y x}\right\}\right] \\
\operatorname{Cov}\left(P_{1 u}, P_{m}\right)= & \bar{Y}^{2}\left[-\frac{1}{N}\left\{C_{y}^{2}+\eta C_{z}^{2}\left(\eta-2 k_{y z}\right)\right\}\right] \\
\operatorname{Cov}\left(P_{2 u}, P_{m}\right)= & \bar{Y}^{2}\left[-\frac{1}{N}\left\{C_{y}^{2}+\eta C_{z}^{2}\left(\eta-2 k_{y z}\right)\right\}\right]
\end{aligned}
$$

PROOF: It is obvious that the $M S E$ of estimators $P_{i}(i=1,2)$ are given by

$$
\begin{aligned}
\operatorname{MSE}\left(P_{i}\right) & =E\left(P_{i}-\bar{Y}\right)^{2} \\
& =E\left[\varphi_{i}\left(P_{i u}-\bar{Y}\right)+\left(1-\varphi_{i}\right)\left(P_{m}-\bar{Y}\right)\right]^{2} \\
& =\varphi_{i}^{2} E\left(P_{i u}-\bar{Y}\right)^{2}+\left(1-\varphi_{i}\right)^{2} E\left(P_{m}-\bar{Y}\right)^{2}+2 \varphi_{i}\left(1-\varphi_{i}\right) E\left[\left(P_{i u}-\bar{Y}\right)\left(P_{m}-\bar{Y}\right)\right] \\
& =\varphi_{i}^{2} \operatorname{MSE}\left(P_{i u}\right)+\left(1-\varphi_{i}\right)^{2} \operatorname{MSE}\left(P_{m}\right)+2 \varphi_{i}\left(1-\varphi_{i}\right) \operatorname{Cov}\left(P_{i u}, P_{m}\right)
\end{aligned}
$$

Substituting the expressions of $P_{1 u}, P_{2 u}$ and $P_{m}$ from (2.5), (2.6) and (2.7) in (2.19), taking expectations and retaining the terms up to the first order of sample sizes, we 
have the expressions for the $M S E$ of the estimators $P_{i}(i=1,2)$ as described in (2.13).

REMARK 2.1: It is assumed that the coefficients of variation and correlation coefficients of non-response class are similar to that of the population, i.e. $C_{y(2)}=C_{y}, C_{z(2)}=C_{z}$ and $\rho_{y z(2)}=\rho_{y z}$. Further since, $x$ and $y$ are the same study variable over two occasions and $z$ is the auxiliary variable correlated to $x$ and $y$, therefore, looking at the stability nature of the coefficient of variation (see, Reddy $(1973,1974)$ and Singh and Ruiz Espejo (2003), the coefficients of variation of the variables $x, y$ and $z$ in the population are considered equal, that is, $C_{x}=C_{y}=C_{z}$.

THEOREM 2.3: Under the Remark 2.1 the bias of the estimator $P_{i}(i=1,2)$ to the first degree of approximation are obtained as

$$
B\left(P_{i}\right)=\varphi_{i} B\left(P_{i u}\right)+\left(1-\varphi_{i}\right) B\left(T_{m}\right),(i=1,2)
$$

where

$$
\begin{aligned}
& B\left(P_{1 u}\right)=\left(\frac{\eta \bar{Y}}{2}\right) C_{y}^{2}\left[\left(\frac{1}{u}-\frac{1}{N}\right)\left(\eta-2 \rho_{y z}+1\right)\right] \\
& B\left(P_{2 u}\right)=\left(\frac{\eta \bar{Y}}{2}\right) C_{y}^{2}\left[\left\{\left(\frac{1}{u}-\frac{1}{N}\right)+\frac{W\left(f_{2}-1\right)}{u}\right\}\left(\eta-2 \rho_{y z}+1\right)\right] \\
& B\left(P_{m}\right)=\left(\frac{\eta \bar{Y}}{2}\right) C_{y}^{2}\left[\left(\frac{1}{m}-\frac{1}{N}\right)\left\{\eta-2 \rho_{y z}+1\right\}+\left(\frac{1}{m}-\frac{1}{n}\right)\left\{1-2 \rho_{y x}+\eta\left(1+2 k_{x z}\right)\right\}\right]
\end{aligned}
$$

Proof is simple so omitted.

THEOREM 2.4: Under the Remark 2.1 MSE of the estimators $P_{i}(i=1,2)$ to the first degree of approximation are obtained as

$$
\operatorname{MSE}\left(P_{i}\right)=\varphi_{i}^{2} \operatorname{MSE}\left(P_{i u}\right)+\left(1-\varphi_{i}\right)^{2} \operatorname{MSE}\left(P_{m}\right)+2 \varphi_{i}\left(1-\varphi_{i}\right) \operatorname{Cov}\left(P_{i u}, P_{m}\right),(i=1,2)
$$

where

$$
\begin{aligned}
& \operatorname{MSE}\left(P_{1 u}\right)=S_{y}^{2}\left[\left(\frac{1}{u}-\frac{1}{N}\right)\left\{1+\eta\left(\eta-2 \rho_{y z}\right)\right\}+\frac{W\left(f_{2}-1\right)}{u}\right] \\
& \operatorname{MSE}\left(P_{2 u}\right)=S_{y}^{2}\left[\left\{\left(\frac{1}{u}-\frac{1}{N}\right)+\frac{W\left(f_{2}-1\right)}{u}\right\}\left\{1+\eta\left(\eta-2 \rho_{y z}\right)\right\}\right]
\end{aligned}
$$




$$
\begin{aligned}
& \operatorname{MSE}\left(P_{m}\right)=S_{y}^{2}\left[\left(\frac{1}{m}-\frac{1}{N}\right)\left\{1+\eta\left(\eta-2 \rho_{y z}\right)\right\}+\left(\frac{1}{m}-\frac{1}{n}\right) \eta\left\{\eta\left(1+2 \rho_{x z}\right)-2 \rho_{y x}\right\}\right] \\
& \operatorname{Cov}\left(P_{1 u}, P_{m}\right)=S_{y}^{2}\left[-\frac{1}{N}\left\{1+\eta\left(\eta-2 \rho_{y z}\right)\right\}\right] \\
& \operatorname{Cov}\left(P_{2 u}, P_{m}\right)=S_{y}^{2}\left[-\frac{1}{N}\left\{1+\eta\left(\eta-2 \rho_{y z}\right)\right\}\right]
\end{aligned}
$$

Proof is simple so omitted.

\subsection{Minimum MSEs of The Estimators $P_{\mathrm{i}}(i=1,2)$}

Since, the MSEs of the estimators $P_{i}(i=1,2)$ in equation (2.13) are functions of unknown constants $\varphi_{\mathrm{i}}(i=1,2)$; therefore, they are minimized with respect to $\varphi_{\mathrm{i}}$ and sub sequentially the optimum values of $\varphi_{\mathrm{i}}$ are obtained as

$$
\begin{aligned}
\varphi_{i_{\text {opt }}} & =\frac{\operatorname{MSE}\left(P_{m}\right)-\operatorname{Cov}\left(P_{i u}, P_{m}\right)}{\operatorname{MSE}\left(P_{i u}\right)+\operatorname{MSE}\left(P_{m}\right)-2 \operatorname{Cov}\left(P_{i u}, P_{m}\right)} ;(i=1,2) \\
\varphi_{1_{\text {opt }}} & =\frac{[C(\eta)-\mu B(\eta)] \mu}{\left[D_{2}+\mu D_{1}+\mu^{2} D_{0}\right]}, \\
\varphi_{2_{\text {opt }}} & =\frac{[C(\eta)-\mu B(\eta)] \mu}{\left[D_{5}+\mu D_{4}+\mu^{2} D_{3}\right]},
\end{aligned}
$$

where $B(\eta)=\eta\left[2 \rho_{y x}-\eta\left(1+2 \rho_{x z}\right)\right], C(\eta)=\left[1-2 \eta \rho_{y z}+\eta^{2}\right]$,

$$
\begin{aligned}
& D_{0}=-B(\eta), D_{1}=-W\left(f_{2}-1\right), D_{2}=C(\eta)+W\left(f_{2}-1\right), \\
& D_{3}=-B(\eta), D_{4}=-W\left(f_{2}-1\right) C(\eta), D_{5}=\left[1+W\left(f_{2}-1\right)\right] C(\eta) .
\end{aligned}
$$

Now substituting the values of $\varphi_{\text {iopt }}$ in (2.13) we get the optimum MSE of $P_{i}$ $(i=1,2)$ as

$$
\operatorname{MSE}\left(P_{i_{o p t}}\right)=\frac{\operatorname{MSE}\left(P_{i u}\right) \operatorname{MSE}\left(P_{m}\right)-\left\{\operatorname{Cov}\left(P_{i u}, P_{m}\right)\right\}^{2}}{\left[\operatorname{MSE}\left(P_{i u}\right)+\operatorname{MSE}\left(P_{m}\right)-2 \operatorname{Cov}\left(P_{i u}, P_{m}\right)\right]} ;(i=1,2)
$$

Further, substituting the values from (2.14) to (2.18) in (2.23), we get the simplified values of $\operatorname{MSE}\left(P_{\text {iopt }}\right)$ which are given as

$$
M S E_{\min .}\left(P_{1}\right)=\frac{S_{y}^{2}}{n} \frac{\left[A_{2}+\mu_{1} A_{1}+\mu_{1}^{2} A_{0}\right]}{\left[D_{2}+\mu_{1} D_{1}+\mu_{1}^{2} D_{0}\right]}
$$




$$
M S E_{\min .}\left(P_{2}\right)=\frac{S_{y}^{2}}{n} \frac{\left[A_{5}+\mu_{2} A_{4}+\mu_{2}^{2} A_{3}\right]}{\left[D_{5}+\mu_{2} D_{4}+\mu_{2}^{2} D_{3}\right]},
$$

where

$$
\begin{aligned}
& A_{0}=f B(\eta) C(\eta), A_{1}=\left[W\left(f_{2}-1\right)\{f C(\eta)-B(\eta)\}-B(\eta) C(\eta)\right] \\
& A_{2}=(1-f) C(\eta)\left[C(\eta)+W\left(f_{2}-1\right)\right] \\
& A_{3}=f B(\eta) C(\eta) \\
& A_{4}=C(\eta)\left[W f\left(f_{2}-1\right) C(\eta)-\left\{1+W\left(f_{2}-1\right)\right\} B(\eta)\right] \\
& A_{5}=\left[\left\{1+W\left(f_{2}-1\right)\right\}(1-f)\{C(\eta)\}^{2}\right] \\
& f=n / N, f_{2}=\left(u_{2} / u_{2 h}\right) .
\end{aligned}
$$

\subsection{Optimum Replacement Policies (ORPs)}

Since the MSEs of the estimators $P_{i}(i=1,2)$ given in equation (2.34) and (2.35) are the function of $\mu_{i}(i=1,2)$, therefore the optimum values of $\mu_{i}$ are determined to estimate the population mean the population mean with maximum precision and lowest cost. To determine the optimum values of $\mu_{i}$, we minimized MSEs of the estimator $P_{i}$ given in equation (2.34) and (2.35) respectively with respect to $\mu_{i}$ which result in quadratic equations in $\mu_{i}$ and the respective solutions of $\mu_{i}$ say $\hat{\mu}_{i}$ $(i=1,2)$ are given below:

$$
\begin{aligned}
& a_{1} \mu^{2}+2 \mu a_{2}+a_{3}=0, \\
& \widehat{\mu}_{1}=\frac{-a_{2} \pm \sqrt{a_{2}^{2}-a_{1} a_{3}}}{a_{1}}, \\
& b_{1} \mu^{2}+2 \mu b_{2}+b_{3}=0 \\
& \widehat{\mu}_{2}=\frac{-b_{2} \pm \sqrt{b_{2}^{2}-b_{1} b_{3}}}{b_{1}},
\end{aligned}
$$

Where $a_{1}=\left(A_{0} D_{1}-D_{0} A_{1}\right), a_{2}=\left(A_{0} D_{2}-D_{0} A_{2}\right), a_{3}=\left(A_{1} D_{2}-D_{1} A_{2}\right)$,

$$
b_{1}=\left(A_{3} D_{4}-D_{3} A_{4}\right), b_{2}=\left(A_{3} D_{5}-D_{3} A_{5}\right), b_{3}=\left(A_{4} D_{5}-D_{4} A_{5}\right) \text {. }
$$

From equations (2.37) and (2.39), it is obvious that real values of $\mu_{i}(i=1,2)$ exists if, the quantities under square roots are greater than or equal to zero for any 
combination of correlations $\rho_{y x}, \rho_{y z}$ and $\rho_{z x}$ which satisfied the conditions in real situations; two real values of $\hat{\mu}_{i}(i=1,2)$ are possible. Hence, while choosing the values of $\hat{\mu}_{i}$, it should be remembered that $0<\hat{\mu}_{i}<1$. All other values of $\hat{\mu}_{i}$ $(i=1,2)$ are inadmissible. Substituting the admissible values of $\hat{\mu}_{i}$ say $\mu_{i}^{(0)}$ from equations (2.37) and (2.39) in to (2.34) and (2.35) respectively, we have the optimum values of $M S E$ s of $P_{\mathrm{i}}(i=1,2)$ which are shown below:

$$
\begin{aligned}
& M S E_{\text {min. }}\left(P_{1 o p t}\right)=\frac{S_{y}^{2}}{n} \frac{\left[A_{2}+\mu_{1}^{(0)} A_{1}+\mu_{1}^{(0) 2} A_{0}\right]}{\left[D_{2}+\mu_{1}^{(0)} D_{1}+\mu_{1}^{(0) 2} D_{0}\right]} \\
& M S E_{\text {min. }}\left(P_{2 o p t}\right)=\frac{S_{y}^{2}}{n} \frac{\left[A_{5}+\mu_{2}^{(0)} A_{4}+\mu_{2}^{(0) 2} A_{3}\right]}{\left[D_{5}+\mu_{2}^{(0)} D_{4}+\mu_{2}^{(0) 2} D_{3}\right]}
\end{aligned}
$$

\section{EFFICIENCY COMPRESSION}

\subsection{Compression with estimators under complete response:}

The percent relative losses (PRLs) in efficiencies of the $P_{i}(i=1,2)$ are obtained with respect to the similar estimator and natural successive sampling estimator when the non-response not observed on any occasions. The estimator $T_{1}$ is defined under the similar circumstances as the estimator as the estimator $P_{i}$ but under complete response, where as $T_{2}$ is the natural successive sampling estimator and they are given as

$$
T_{j}=\psi_{j} T_{j u}+\left(1-\psi_{j}\right) T_{j m},(j=1,2)
$$

i.e.

$$
\begin{aligned}
& T_{1}=\psi_{1} T_{1 u}+\left(1-\psi_{1}\right) T_{1 m}, \\
& T_{2}=\psi_{2} T_{2 u}+\left(1-\psi_{2}\right) T_{2 m},
\end{aligned}
$$

where

$$
T_{1 u}=\bar{y}_{u}\left(\frac{\bar{Z}}{\bar{z}_{u}}\right)^{\eta}, T_{1 m}=\bar{y}_{m}\left(\frac{\bar{x}_{n}}{\bar{x}_{m}}\right)^{\eta}\left(\frac{\bar{Z}}{\bar{z}_{u}}\right)^{\eta}, T_{2 u}=\bar{y}_{u}, T_{2 m}=\bar{y}_{m}+b_{y x(m)}\left(\bar{x}_{n}-\bar{x}_{m}\right) ;
$$


$T_{1 m}$ is same as $T_{m}$ defined in Sub-section 2.1 and $b_{y x}$ is the simple regression coefficients between the variables shown in suffices. Proceeding in the similar line as discussed for the estimators $P_{\mathrm{i}}(i=1,2)$, the optimum MSE of the estimators $T_{j}$ $(j=1,2)$ are derived as

$$
\begin{aligned}
& M S E_{\text {min. }}\left(T_{1 o p t}\right)=\frac{S_{y}^{2}}{n} \frac{\left[A_{8}+\mu_{3}^{(0)} A_{7}+\mu_{3}^{(0) 2} A_{6}\right]}{\left[D_{7}+\mu_{3}^{(0) 2} D_{6}\right]} \\
& M S E_{\text {min. }}\left(T_{2 o p t}\right)=\frac{S_{y}^{2}}{n}\left[\frac{1}{2}\left\{1+\sqrt{\left(1-\rho_{y x}^{2}\right)}\right\}-f\right]
\end{aligned}
$$

where $\hat{\mu}_{3}=\frac{C(\eta) \pm \sqrt{A(\eta) C(\eta)}}{B(\eta)}$, and $\mu_{3}^{(0)}$ is the admissible value obtained from $\hat{\mu}_{3}$;

$f=n / N$ :fraction of the fresh sample for the estimator;

$$
\begin{aligned}
& D_{7}=C(\eta), D_{6}=-B(\eta), A_{8}=(1-f)\{C(\eta)\}^{2}, A_{7}=-B(\eta) C(\eta), \\
& A_{6}=f B(\eta) C(\eta) .
\end{aligned}
$$

REMARKS 3.1: To compare the performance of the estimators $P_{i}(i=1,2)$ with the estimators $T_{j}(j=1,2)$, we introduce an assumption $\rho_{y z}=\rho_{x z}$, which is an intuitive assumption and also considered by Cochran (1977) and Feng and Zou (1997).

The PRLs in precision of estimators $P_{\mathrm{i}}(i=1,2)$ with respect to $T_{j}(j=1,2)$ under their respective optimality conditions are given as

$$
L_{i j}=\frac{M S E_{\text {min. }}\left(P_{i o p t}\right)-M S E_{\text {min. }}\left(T_{\text {jopt }}\right)}{M S E_{\text {min. }}\left(P_{i o p t}\right)} \times 100 ;(i, j=1,2)
$$

For $N=5000, n=500$ and $f_{2}=1.5$ for different choices of $\rho_{y x}, \rho_{y z}$ and $\rho_{x z}$, Table 3.1 to 3.4 give the optimum values of $\mu_{i}^{(0)}$ and PRLs in precisions $L_{i j}(i, j=1,2)$ of estimators $P_{i}(i=1,2)$ with respect to estimators $T_{j}(j=1,2)$. 
3.2. Compression with Hansen and Hurwitz (1946) estimator under nonresponse:

The recent relative losses in precision of estimators $P_{i}(i=1,2)$ with respect to Hansen and Hurwitz (1946) estimator $\bar{y}_{n}^{*}$, when non-response occurs at current occasion and when there is no matching from the previous occasion:

$$
\bar{y}_{n}^{*}=\frac{n_{1} \bar{y}_{n 1}+n_{2} \bar{y}_{n 2 h}}{n}
$$

Since, $\bar{y}_{n}^{*}$ is an unbiased estimator of $\bar{Y}$; therefore, following Sukhatme et al. (1984) the variance of $\bar{y}_{n}^{*}$ id given as:

$$
\operatorname{Var}\left(\bar{y}_{n}^{*}\right)=\frac{S_{y}^{2}}{n}\left[(1-f)+W\left(f_{2}^{*}-1\right)\right]
$$

The expression of the variance in (3.8) is written under the assumption $S_{y}^{2}=S_{y(2)}^{2}$.

REMARKS 3.2: To compare the performance of the estimators $P_{\mathrm{i}}(i=1,2)$ with respect to Hansen and Hurwitz (1946) estimators $\bar{y}_{n}^{*}$, we introduce one more assumption $f_{2}=f_{2}^{*}$.

The PRLs in precision of estimators $P_{i}(i=1,2)$ with respect to Hansen and Hurwitz (1946) estimator $\bar{y}_{n}^{*}$ under their respective optimality conditions are given as

$$
L_{i}=\frac{M S E_{\text {min. }}\left(P_{\text {iopt }}\right)-\operatorname{Var}\left(\bar{y}_{n}^{*}\right)}{M S E_{\text {min. }}\left(P_{\text {iopt }}\right)} \times 100 ;(i=1,2)
$$

For $N=5000, n=500$ and $f_{2}=1.5$ for different choices of $\rho_{y x}, \rho_{y z}$ and $\rho_{x z}$, Table 3.5 and 3.6 give the optimum values of $\mu_{i}^{(0)}$ and PRLs in precisions $L_{\mathrm{i}}(i=1,2)$ of estimators $P_{i}(i=1,2)$ with respect to Hansen and Hurwitz (1946) estimator $\bar{y}_{n}^{*}$.

NOTE: For the sake brevity and to save the space we have only given the values of $L_{i j}(i, j=1,2)$ and $L_{i}(i=1,2)$ given by (3.6) and (3.9) respectively for $\eta=0.25$. For other values of $\eta$ like $0.50,0.75$ and 1.00 the values $L_{\mathrm{ij}}(i, j=1,2)$ and $L_{i}$ $(i=1,2)$ are with the first author of the paper. 
JAMSI, 13 (2017), No. 2 
Table 3.1: The $P R L s$ in precision $L_{11}$ of the estimator $P_{1}$ with respect to $T_{1}$ at $\eta=0.25$

\begin{tabular}{|c|c|c|c|c|c|c|c|c|c|c|}
\hline \multicolumn{3}{|c|}{$\rho_{x z}$} & \multicolumn{2}{|c|}{0.5} & \multicolumn{2}{|c|}{0.6} & \multicolumn{2}{|c|}{0.7} & \multicolumn{2}{|c|}{0.8} \\
\hline$W$ & $\rho_{y x}$ & $\rho_{y z}$ & $\mu_{1}^{(0)}$ & $L_{11}$ & $\mu_{1}^{(0)}$ & $L_{11}$ & $\mu_{1}^{(0)}$ & $L_{11}$ & $\mu_{1}^{(0)}$ & $L_{11}$ \\
\hline \multirow{20}{*}{0.05} & \multirow{5}{*}{0.5} & 0.5 & 0.4296 & 1.5188 & 0.4163 & 1.4999 & 0.4002 & 1.4764 & 0.3803 & 1.4465 \\
\hline & & 0.6 & 0.4317 & 1.6171 & 0.4182 & 1.5969 & 0.4020 & 1.5719 & 0.3819 & 1.5401 \\
\hline & & 0.7 & 0.4341 & 1.7290 & 0.4204 & 1.7074 & 0.4040 & 1.6807 & 0.3837 & 1.6466 \\
\hline & & 0.8 & 0.4369 & 1.8576 & 0.4229 & 1.8343 & 0.4063 & 1.8056 & 0.3858 & 1.7690 \\
\hline & & 0.9 & 0.4402 & 2.0069 & 0.4260 & 1.9817 & 0.4091 & 1.9506 & 0.3883 & 1.9111 \\
\hline & \multirow{5}{*}{0.6} & 0.5 & 0.4675 & 1.5689 & 0.4596 & 1.5590 & 0.4508 & 1.5477 & 0.4409 & 1.5345 \\
\hline & & 0.6 & 0.4704 & 1.6706 & 0.4622 & 1.6601 & 0.4533 & 1.6479 & 0.4432 & 1.6338 \\
\hline & & 0.7 & 0.4737 & 1.7865 & 0.4653 & 1.7751 & 0.4561 & 1.7621 & 0.4458 & 1.7470 \\
\hline & & 0.8 & 0.4776 & 1.9197 & 0.4689 & 1.9074 & 0.4594 & 1.8933 & 0.4489 & 1.8769 \\
\hline & & 0.9 & 0.4822 & 2.0744 & 0.4732 & 2.0610 & 0.4634 & 2.0456 & 0.4525 & 2.0279 \\
\hline & \multirow{5}{*}{0.7} & 0.5 & 0.4936 & 1.5986 & 0.4877 & 1.5923 & 0.4814 & 1.5853 & 0.4747 & 1.5776 \\
\hline & & 0.6 & 0.4973 & 1.7026 & 0.4912 & 1.6958 & 0.4847 & 1.6883 & 0.4778 & 1.6800 \\
\hline & & 0.7 & 0.5017 & 1.8211 & 0.4953 & 1.8137 & 0.4885 & 1.8056 & 0.4814 & 1.7966 \\
\hline & & 0.8 & 0.5070 & 1.9574 & 0.5002 & 1.9493 & 0.4931 & 1.9404 & 0.4856 & 1.9306 \\
\hline & & 0.9 & 0.5132 & 2.1158 & 0.5060 & 2.1069 & 0.4985 & 2.0971 & 0.4906 & 2.0864 \\
\hline & \multirow{5}{*}{0.8} & 0.5 & 0.5148 & 1.6193 & 0.5097 & 1.6147 & 0.5045 & 1.6097 & 0.4992 & 1.6044 \\
\hline & & 0.6 & 0.5196 & 1.7250 & 0.5143 & 1.7200 & 0.5088 & 1.7146 & 0.5032 & 1.7088 \\
\hline & & 0.7 & 0.5254 & 1.8455 & 0.5197 & 1.8400 & 0.5139 & 1.8342 & 0.5079 & 1.8279 \\
\hline & & 0.8 & 0.5322 & 1.9844 & 0.5261 & 1.9782 & 0.5199 & 1.9717 & 0.5135 & 1.9648 \\
\hline & & 0.9 & 0.5405 & 2.1459 & 0.5339 & 2.1391 & 0.5271 & 2.1318 & 0.5203 & 2.1241 \\
\hline \multirow{20}{*}{0.10} & \multirow{5}{*}{0.5} & 0.5 & 0.3396 & 2.6737 & 0.3154 & 2.6029 & 0.2858 & 2.5149 & 0.2484 & 2.4022 \\
\hline & & 0.6 & 0.3424 & 2.8449 & 0.3180 & 2.7698 & 0.2882 & 2.6764 & 0.2507 & 2.5569 \\
\hline & & 0.7 & 0.3456 & 3.0396 & 0.3210 & 2.9596 & 0.2910 & 2.8602 & 0.2533 & 2.7330 \\
\hline & & 0.8 & 0.3492 & 3.2630 & 0.3245 & 3.1774 & 0.2943 & 3.0711 & 0.2564 & 2.9351 \\
\hline & & 0.9 & 0.3536 & 3.5218 & 0.3285 & 3.4298 & 0.2981 & 3.3155 & 0.2599 & 3.1695 \\
\hline & \multirow{5}{*}{0.6} & 0.5 & 0.4056 & 2.8583 & 0.3923 & 2.8224 & 0.3772 & 2.7807 & 0.3599 & 2.7319 \\
\hline & & 0.6 & 0.4091 & 3.0412 & 0.3956 & 3.0029 & 0.3803 & 2.9586 & 0.3628 & 2.9067 \\
\hline & & 0.7 & 0.4132 & 3.2490 & 0.3994 & 3.2081 & 0.3839 & 3.1609 & 0.3662 & 3.1055 \\
\hline & & 0.8 & 0.4179 & 3.4875 & 0.4039 & 3.4436 & 0.3881 & 3.3929 & 0.3701 & 3.3336 \\
\hline & & 0.9 & 0.4236 & 3.7638 & 0.4092 & 3.7164 & 0.3931 & 3.6617 & 0.3748 & 3.5978 \\
\hline & \multirow{5}{*}{0.7} & 0.5 & 0.4474 & 2.9646 & 0.4382 & 2.9424 & 0.4284 & 2.9176 & 0.4176 & 2.8898 \\
\hline & & 0.6 & 0.4518 & 3.1545 & 0.4424 & 3.1307 & 0.4323 & 3.1043 & 0.4213 & 3.0746 \\
\hline & & 0.7 & 0.4569 & 3.3705 & 0.4473 & 3.3449 & 0.4369 & 3.3166 & 0.4256 & 3.2848 \\
\hline & & 0.8 & 0.4630 & 3.6183 & 0.4530 & 3.5907 & 0.4423 & 3.5601 & 0.4306 & 3.5260 \\
\hline & & 0.9 & 0.4703 & 3.9058 & 0.4599 & 3.8757 & 0.4487 & 3.8425 & 0.4367 & 3.8055 \\
\hline & \multirow{5}{*}{0.8} & 0.5 & 0.4786 & 3.0357 & 0.4714 & 3.0201 & 0.4639 & 3.0032 & 0.4559 & 2.9848 \\
\hline & & 0.6 & 0.4841 & 3.2307 & 0.4767 & 3.2139 & 0.4688 & 3.1958 & 0.4606 & 3.1761 \\
\hline & & 0.7 & 0.4906 & 3.4526 & 0.4828 & 3.4345 & 0.4746 & 3.4149 & 0.4660 & 3.3937 \\
\hline & & 0.8 & 0.4984 & 3.7076 & 0.4901 & 3.6878 & 0.4815 & 3.6665 & 0.4725 & 3.6435 \\
\hline & & 0.9 & 0.5078 & 4.0036 & 0.4989 & 3.9818 & 0.4898 & 3.9584 & 0.4803 & 3.9332 \\
\hline
\end{tabular}




\begin{tabular}{|c|c|c|c|c|c|c|c|c|c|c|}
\hline \multicolumn{3}{|c|}{$\rho_{y z}$} & \multicolumn{2}{|c|}{0.5} & \multicolumn{2}{|c|}{0.6} & \multicolumn{2}{|c|}{0.7} & \multicolumn{2}{|c|}{0.8} \\
\hline$W$ & $\rho_{y x}$ & $\rho_{y z}$ & $\mu_{1}^{(0)}$ & $L_{11}$ & $\mu_{1}^{(0)}$ & $L_{11}$ & $\mu_{1}^{(0)}$ & $L_{11}$ & $\mu_{1}^{(0)}$ & $L_{11}$ \\
\hline \multirow{20}{*}{0.15} & \multirow{5}{*}{0.5} & 0.5 & 0.2510 & 3.5089 & 0.2161 & 3.3578 & 0.1730 & 3.1694 & 0.1183 & 2.9279 \\
\hline & & 0.6 & 0.2545 & 3.7334 & 0.2194 & 3.5735 & 0.1762 & 3.3744 & 0.1215 & 3.1191 \\
\hline & & 0.7 & 0.2585 & 3.9886 & 0.2232 & 3.8189 & 0.1799 & 3.6076 & 0.1250 & 3.3368 \\
\hline & & 0.8 & 0.2631 & 4.2811 & 0.2277 & 4.1004 & 0.1842 & 3.8754 & 0.1291 & 3.5871 \\
\hline & & 0.9 & 0.2686 & 4.6201 & 0.2329 & 4.4266 & 0.1891 & 4.1860 & 0.1339 & 3.8778 \\
\hline & \multirow{5}{*}{0.6} & 0.5 & 0.3447 & 3.9014 & 0.3260 & 3.8253 & 0.3047 & 3.7369 & 0.2801 & 3.6330 \\
\hline & & 0.6 & 0.3489 & 4.1490 & 0.3300 & 4.0683 & 0.3085 & 3.9747 & 0.2837 & 3.8647 \\
\hline & & 0.7 & 0.3537 & 4.4301 & 0.3346 & 4.3443 & 0.3129 & 4.2448 & 0.2879 & 4.1280 \\
\hline & & 0.8 & 0.3594 & 4.7522 & 0.3400 & 4.6606 & 0.3181 & 4.5544 & 0.2928 & 4.4298 \\
\hline & & 0.9 & 0.3661 & 5.1250 & 0.3464 & 5.0266 & 0.3241 & 4.9128 & 0.2985 & 4.7792 \\
\hline & \multirow{5}{*}{0.7} & 0.5 & 0.4018 & 4.1249 & 0.3895 & 4.0783 & 0.3761 & 4.0263 & 0.3612 & 3.9678 \\
\hline & & 0.6 & 0.4069 & 4.3861 & 0.3944 & 4.3366 & 0.3807 & 4.2814 & 0.3656 & 4.2194 \\
\hline & & 0.7 & 0.4129 & 4.6829 & 0.4000 & 4.6300 & 0.3860 & 4.5712 & 0.3707 & 4.5050 \\
\hline & & 0.8 & 0.4199 & 5.0229 & 0.4067 & 4.9662 & 0.3923 & 4.9031 & 0.3767 & 4.8324 \\
\hline & & 0.9 & 0.4282 & 5.4165 & 0.4146 & 5.3553 & 0.3998 & 5.2873 & 0.3838 & 5.2111 \\
\hline & \multirow{5}{*}{0.8} & 0.5 & 0.4430 & 4.2722 & 0.4337 & 4.2401 & 0.4238 & 4.2052 & 0.4132 & 4.1669 \\
\hline & & 0.6 & 0.4492 & 4.5431 & 0.4396 & 4.5088 & 0.4294 & 4.4716 & 0.4186 & 4.4309 \\
\hline & & 0.7 & 0.4565 & 4.8509 & 0.4465 & 4.8141 & 0.4360 & 4.7742 & 0.4248 & 4.7307 \\
\hline & & 0.8 & 0.4651 & 5.2040 & 0.4547 & 5.1642 & 0.4438 & 5.1212 & 0.4322 & 5.0743 \\
\hline & & 0.9 & 0.4757 & 5.6130 & 0.4646 & 5.5697 & 0.4531 & 5.5229 & 0.4410 & 5.4721 \\
\hline \multirow{20}{*}{0.20} & \multirow{5}{*}{0.5} & 0.5 & 0.1636 & 4.0625 & 0.1181 & 3.8060 & 0.0618 & 3.4862 & -0.0099 & 3.0756 \\
\hline & & 0.6 & 0.1679 & 4.3250 & 0.1222 & 4.0545 & 0.0659 & 3.7172 & -0.0059 & 3.2843 \\
\hline & & 0.7 & 0.1728 & 4.6236 & 0.1270 & 4.3374 & 0.0705 & 3.9807 & -0.0013 & 3.5230 \\
\hline & & 0.8 & 0.1785 & 4.9663 & 0.1325 & 4.6625 & 0.0759 & 4.2840 & 0.0040 & 3.7984 \\
\hline & & 0.9 & 0.1851 & 5.3636 & 0.1390 & 5.0399 & 0.0822 & 4.6368 & 0.0102 & 4.1197 \\
\hline & \multirow{5}{*}{0.6} & 0.5 & 0.2845 & 4.7267 & 0.2606 & 4.5981 & 0.2332 & 4.4487 & 0.2013 & 4.2729 \\
\hline & & 0.6 & 0.2895 & 5.0260 & 0.2654 & 4.8902 & 0.2378 & 4.7325 & 0.2058 & 4.5469 \\
\hline & & 0.7 & 0.2952 & 5.3659 & 0.2708 & 5.2220 & 0.2431 & 5.0550 & 0.2108 & 4.8585 \\
\hline & & 0.8 & 0.3018 & 5.7551 & 0.2772 & 5.6020 & 0.2492 & 5.4245 & 0.2167 & 5.2157 \\
\hline & & 0.9 & 0.3096 & 6.2053 & 0.2847 & 6.0417 & 0.2563 & 5.8522 & 0.2236 & 5.6295 \\
\hline & \multirow{5}{*}{0.7} & 0.5 & 0.3570 & 5.1026 & 0.3415 & 5.0244 & 0.3245 & 4.9371 & 0.3057 & 4.8386 \\
\hline & & 0.6 & 0.3628 & 5.4236 & 0.3471 & 5.3408 & 0.3298 & 5.2484 & 0.3108 & 5.1443 \\
\hline & & 0.7 & 0.3695 & 5.7878 & 0.3535 & 5.6999 & 0.3360 & 5.6017 & 0.3167 & 5.4913 \\
\hline & & 0.8 & 0.3774 & 6.2048 & 0.3611 & 6.1109 & 0.3433 & 6.0063 & 0.3236 & 5.8886 \\
\hline & & 0.9 & 0.3869 & 6.6869 & 0.3701 & 6.5862 & 0.3518 & 6.4740 & 0.3318 & 6.3480 \\
\hline & \multirow{5}{*}{0.8} & 0.5 & 0.4079 & 5.3484 & 0.3965 & 5.2950 & 0.3843 & 5.2368 & 0.3711 & 5.1730 \\
\hline & & 0.6 & 0.4148 & 5.6844 & 0.4031 & 5.6276 & 0.3906 & 5.5658 & 0.3772 & 5.4982 \\
\hline & & 0.7 & 0.4229 & 6.0657 & 0.4108 & 6.0051 & 0.3980 & 5.9393 & 0.3843 & 5.8672 \\
\hline & & 0.8 & 0.4325 & 6.5024 & 0.4199 & 6.4374 & 0.4067 & 6.3668 & 0.3926 & 6.2896 \\
\hline & & 0.9 & 0.4441 & 7.0078 & 0.4310 & 6.9374 & 0.4171 & 6.8612 & 0.4025 & 6.7781 \\
\hline
\end{tabular}


Table 3.2: The PRLs in precision $L_{12}$ of the estimator $P_{1}$ with respect to $T_{2}$ at $\eta=0.25$

\begin{tabular}{|c|c|c|c|c|c|c|c|c|c|c|}
\hline \multicolumn{3}{|l|}{$\rho_{x z}$} & \multicolumn{2}{|l|}{0.5} & \multicolumn{2}{|l|}{0.6} & \multicolumn{2}{|l|}{0.7} & \multicolumn{2}{|l|}{0.8} \\
\hline$W$ & $\rho_{y x}$ & $\rho_{y z}$ & $\mu_{1}^{(0)}$ & $L_{12}$ & $\mu_{1}^{(0)}$ & $L_{12}$ & $\mu_{1}^{(0)}$ & $L_{12}$ & $\mu_{1}^{(0)}$ & $L_{12}$ \\
\hline \multirow{20}{*}{0.05} & \multirow{5}{*}{0.5} & 0.5 & 0.4296 & -17.4132 & 0.4163 & -16.8701 & 0.4002 & -16.3425 & 0.3803 & -15.8322 \\
\hline & & \begin{tabular}{|l|}
0.6 \\
\end{tabular} & 0.4317 & \begin{tabular}{|l|}
-25.3885 \\
\end{tabular} & \begin{tabular}{|l|}
0.4182 \\
\end{tabular} & \begin{tabular}{|l|}
-24.7650 \\
\end{tabular} & 0.4020 & \begin{tabular}{|l|}
-24.1601 \\
\end{tabular} & 0.3819 & -23.5758 \\
\hline & & 0.7 & 0.4341 & -34.5304 & 0.4204 & -33.8072 & 0.4040 & -33.1067 & 0.3837 & -32.4310 \\
\hline & & 0.8 & 0.4369 & -45.1163 & 0.4229 & -44.2674 & 0.4063 & -43.4465 & 0.3858 & -42.6561 \\
\hline & & 0.9 & 0.4402 & -57.5194 & 0.4260 & -56.5088 & 0.4091 & -55.5336 & 0.3883 & -54.5966 \\
\hline & \multirow{5}{*}{0.6} & 0.5 & 0.4675 & -14.9812 & 0.4596 & -14.4062 & 0.4508 & -13.8443 & 0.4409 & -13.2955 \\
\hline & & 0.6 & 0.4704 & -22.9785 & 0.4622 & -22.3145 & 0.4533 & -21.6667 & 0.4432 & -21.0349 \\
\hline & & \begin{tabular}{|l|l|}
0.7 \\
\end{tabular} & 0.4737 & -32.1801 & 0.4653 & -31.4046 & 0.4561 & -30.6493 & 0.4458 & -29.9140 \\
\hline & & 0.8 & 0.4776 & -42.8826 & 0.4689 & -41.9647 & 0.4594 & -41.0728 & 0.4489 & -40.2063 \\
\hline & & 0.9 & 0.4822 & -55.4903 & 0.4732 & -54.3866 & 0.4634 & \begin{tabular}{|l|}
-53.3169 \\
\end{tabular} & 0.4525 & -52.2805 \\
\hline & \multirow{5}{*}{0.7} & 0.5 & 0.4936 & -11.1147 & 0.4877 & -10.5192 & 0.4814 & -9.9370 & 0.4747 & -9.3678 \\
\hline & & 0.6 & 0.4973 & -19.0509 & 0.4912 & -18.3585 & 0.4847 & -17.6827 & 0.4778 & -17.0232 \\
\hline & & \begin{tabular}{|l|}
0.7 \\
\end{tabular} & 0.5017 & -28.2234 & 0.4953 & -27.4080 & 0.4885 & -26.6139 & 0.4814 & -25.8405 \\
\hline & & 0.8 & 0.5070 & -38.9503 & 0.5002 & -37.9755 & 0.4931 & -37.0289 & 0.4856 & -36.1092 \\
\hline & & 0.9 & 0.5132 & -51.6719 & 0.5060 & -50.4853 & 0.4985 & -49.3367 & 0.4906 & -48.2243 \\
\hline & \multirow{5}{*}{0.8} & 0.5 & 0.5148 & \begin{tabular}{|l|}
-5.0718 \\
\end{tabular} & 0.5097 & -4.4678 & 0.5045 & -3.8779 & 0.4992 & -3.3016 \\
\hline & & \begin{tabular}{|l|}
0.6 \\
\end{tabular} & 0.5196 & -12.8035 & 0.5143 & -12.0954 & 0.5088 & -11.4054 & 0.5032 & -10.7326 \\
\hline & & 0.7 & 0.5254 & -21.7886 & 0.5197 & -20.9465 & 0.5139 & -20.1280 & 0.5079 & -19.3319 \\
\hline & & 0.8 & 0.5322 & -32.3674 & 0.5261 & -31.3485 & 0.5199 & -30.3613 & 0.5135 & -29.4042 \\
\hline & & 0.9 & 0.5405 & -45.0206 & 0.5339 & -43.7612 & 0.5271 & -42.5460 & 0.5203 & -41.3725 \\
\hline \multirow{20}{*}{0.10} & \multirow{5}{*}{0.5} & 0.5 & 0.3396 & -16.0363 & 0.3154 & -15.5613 & 0.2858 & -15.1162 & 0.2484 & -14.7090 \\
\hline & & 0.6 & 0.3424 & -23.8237 & 0.3180 & -23.2779 & 0.2882 & -22.7669 & 0.2507 & -22.2996 \\
\hline & & \begin{tabular}{|l|}
0.7 \\
\end{tabular} & 0.3456 & \begin{tabular}{|l|}
-32.7362 \\
\end{tabular} & 0.3210 & -32.1026 & 0.2910 & \begin{tabular}{|l|}
-31.5098 \\
\end{tabular} & 0.2533 & -30.9682 \\
\hline & & 0.8 & 0.3492 & -43.0382 & 0.3245 & -42.2936 & 0.2943 & -41.5978 & 0.2564 & -40.9626 \\
\hline & & 0.9 & 0.3536 & -55.0843 & 0.3285 & -54.1966 & 0.2981 & -53.3685 & 0.2599 & -52.6132 \\
\hline & \multirow{5}{*}{0.6} & 0.5 & 0.4056 & -13.4749 & 0.3923 & -12.9380 & 0.3772 & -12.4185 & 0.3599 & -11.9177 \\
\hline & & 0.6 & 0.4091 & -21.2644 & 0.3956 & -20.6443 & 0.3803 & -20.0453 & 0.3628 & -19.4686 \\
\hline & & \begin{tabular}{|l|}
0.7 \\
\end{tabular} & 0.4132 & -30.2118 & 0.3994 & -29.4875 & 0.3839 & -28.7890 & 0.3662 & -28.1177 \\
\hline & & 0.8 & 0.4179 & -40.5987 & 0.4039 & -39.7415 & 0.3881 & -38.9165 & 0.3701 & -38.1250 \\
\hline & & 0.9 & 0.4236 & -52.8079 & 0.4092 & -51.7772 & 0.3931 & -50.7875 & 0.3748 & -49.8403 \\
\hline & \multirow{5}{*}{0.7} & 0.5 & 0.4474 & -9.5722 & 0.4382 & -9.0030 & 0.4284 & -8.4488 & 0.4176 & $\begin{array}{l}-7.9097 \\
\end{array}$ \\
\hline & & 0.6 & 0.4518 & -17.2925 & 0.4424 & \begin{tabular}{|l|}
-16.6308 \\
\end{tabular} & 0.4323 & \begin{tabular}{|l|}
-15.9877 \\
\end{tabular} & 0.4213 & -15.3632 \\
\hline & & \begin{tabular}{|l|}
0.7 \\
\end{tabular} & 0.4569 & \begin{tabular}{|l|}
-26.1999 \\
\end{tabular} & \begin{tabular}{|l|}
0.4473 \\
\end{tabular} & -25.4210 & 0.4369 & -24.6656 & 0.4256 & -23.9334 \\
\hline & & \begin{tabular}{|l|}
0.8 \\
\end{tabular} & 0.4630 & -36.5964 & 0.4530 & -35.6657 & 0.4423 & \begin{tabular}{|l|}
-34.7655 \\
\end{tabular} & 0.4306 & -33.8950 \\
\hline & & 0.9 & 0.4703 & -48.8984 & 0.4599 & -47.7662 & 0.4487 & -46.6744 & 0.4367 & -45.6219 \\
\hline & \multirow{5}{*}{0.8} & 0.5 & 0.4786 & -3.5590 & 0.4714 & -2.9755 & 0.4639 & -2.4067 & 0.4559 & -1.8524 \\
\hline & & 0.6 & 0.4841 & -11.0752 & 0.4767 & -10.3914 & 0.4688 & -9.7264 & 0.4606 & -9.0796 \\
\hline & & \begin{tabular}{|l|}
0.7 \\
\end{tabular} & 0.4906 & -19.7945 & 0.4828 & -18.9819 & 0.4746 & -18.1935 & 0.4660 & -17.4286 \\
\hline & & 0.8 & 0.4984 & -30.0403 & 0.4901 & -29.0577 & 0.4815 & \begin{tabular}{|l|}
-28.1075 \\
\end{tabular} & 0.4725 & -27.1884 \\
\hline & & 0.9 & 0.5078 & -42.2676 & 0.4989 & -41.0541 & 0.4898 & -39.8855 & 0.4803 & -38.7594 \\
\hline
\end{tabular}


JAMSI, 13 (2017), No. 2

\begin{tabular}{|c|c|c|c|c|c|c|c|c|c|c|}
\hline \multicolumn{3}{|c|}{$\rho_{y z}$} & \multicolumn{2}{|c|}{0.5} & \multicolumn{2}{|c|}{0.6} & \multicolumn{2}{|c|}{0.7} & \multicolumn{2}{|c|}{0.8} \\
\hline$W$ & $\rho_{y x}$ & $\rho_{y z}$ & $\mu_{1}^{(0)}$ & $L_{12}$ & $\mu_{1}^{(0)}$ & $L_{12}$ & $\mu_{1}^{(0)}$ & $L_{12}$ & $\mu_{1}^{(0)}$ & $L_{12}$ \\
\hline \multirow{20}{*}{0.15} & \multirow{5}{*}{0.5} & 0.5 & 0.2510 & -15.0405 & 0.2161 & -14.6657 & 0.1730 & -14.3432 & 0.1183 & -14.0910 \\
\hline & & 0.6 & 0.2545 & -22.6913 & 0.2194 & -22.2589 & 0.1762 & -21.8865 & 0.1215 & -21.5941 \\
\hline & & 0.7 & 0.2585 & -31.4372 & 0.2232 & -30.9329 & 0.1799 & -30.4980 & 0.1250 & -30.1552 \\
\hline & & 0.8 & 0.2631 & -41.5328 & 0.2277 & -40.9371 & 0.1842 & -40.4229 & 0.1291 & -40.0158 \\
\hline & & 0.9 & 0.2686 & -53.3189 & 0.2329 & -52.6048 & 0.1891 & -51.9877 & 0.1339 & -51.4968 \\
\hline & \multirow{5}{*}{0.6} & 0.5 & 0.3447 & -12.2565 & 0.3260 & -11.7724 & 0.3047 & -11.3128 & 0.2801 & -10.8809 \\
\hline & & 0.6 & 0.3489 & -19.8789 & 0.3300 & -19.3192 & 0.3085 & -18.7883 & 0.2837 & -18.2898 \\
\hline & & 0.7 & 0.3537 & -28.6222 & 0.3346 & -27.9676 & 0.3129 & -27.3474 & 0.2879 & -26.7657 \\
\hline & & 0.8 & 0.3594 & -38.7562 & 0.3400 & -37.9802 & 0.3181 & -37.2462 & 0.2928 & -36.5586 \\
\hline & & 0.9 & 0.3661 & -50.6464 & 0.3464 & -49.7117 & 0.3241 & -48.8293 & 0.2985 & -48.0039 \\
\hline & \multirow{5}{*}{0.7} & 0.5 & 0.4018 & -8.2620 & 0.3895 & -7.7272 & 0.3761 & -7.2103 & 0.3612 & -6.7117 \\
\hline & & 0.6 & 0.4069 & -15.8008 & 0.3944 & -15.1789 & 0.3807 & -14.5786 & 0.3656 & -14.0007 \\
\hline & & 0.7 & 0.4129 & -24.4858 & 0.4000 & -23.7534 & 0.3860 & -23.0479 & 0.3707 & -22.3698 \\
\hline & & 0.8 & 0.4199 & -34.6057 & 0.4067 & -33.7302 & 0.3923 & -32.8888 & 0.3767 & -32.0819 \\
\hline & & 0.9 & 0.4282 & -46.5574 & 0.4146 & -45.4916 & 0.3998 & -44.4705 & 0.3838 & -43.4940 \\
\hline & \multirow{5}{*}{0.8} & 0.5 & 0.4430 & -2.2384 & 0.4337 & -1.6801 & 0.4238 & -1.1377 & 0.4132 & -0.6113 \\
\hline & & 0.6 & 0.4492 & -9.5688 & 0.4396 & -8.9146 & 0.4294 & -8.2804 & 0.4186 & -7.6660 \\
\hline & & 0.7 & 0.4565 & -18.0595 & 0.4465 & -17.2820 & 0.4360 & -16.5301 & 0.4248 & -15.8034 \\
\hline & & 0.8 & 0.4651 & -28.0195 & 0.4547 & -27.0793 & 0.4438 & -26.1731 & 0.4322 & -25.2998 \\
\hline & & 0.9 & 0.4757 & -39.8824 & 0.4646 & -38.7214 & 0.4531 & -37.6068 & 0.4410 & -36.5365 \\
\hline \multirow{20}{*}{0.20} & \multirow{5}{*}{0.5} & 0.5 & 0.1636 & -14.3805 & 0.1181 & -14.1338 & 0.0618 & -13.9692 & $*$ & - \\
\hline & & 0.6 & 0.1679 & -21.9374 & 0.1222 & -21.6491 & 0.0659 & -21.4540 & $*$ & - \\
\hline & & 0.7 & 0.1728 & -30.5679 & 0.1270 & -30.2270 & 0.0705 & -29.9929 & $*$ & - \\
\hline & & 0.8 & 0.1785 & -40.5197 & 0.1325 & -40.1109 & 0.0759 & -39.8259 & 0.0040 & -39.7089 \\
\hline & & 0.9 & 0.1851 & -52.1237 & 0.1390 & -51.6255 & 0.0822 & -51.2726 & 0.0102 & -51.1156 \\
\hline & \multirow{5}{*}{0.6} & 0.5 & 0.2845 & -11.2925 & 0.2606 & -10.8742 & 0.2332 & -10.4897 & 0.2013 & -10.1447 \\
\hline & & 0.6 & 0.2895 & -18.7820 & 0.2654 & -18.2969 & 0.2378 & -17.8509 & 0.2058 & -17.4504 \\
\hline & & 0.7 & 0.2952 & -27.3628 & 0.2708 & -26.7934 & 0.2431 & -26.2701 & 0.2108 & -25.7999 \\
\hline & & 0.8 & 0.3018 & -37.2953 & 0.2772 & -36.6177 & 0.2492 & -35.9951 & 0.2167 & -35.4356 \\
\hline & & 0.9 & 0.3096 & -48.9312 & 0.2847 & -48.1115 & 0.2563 & -47.3589 & 0.2236 & -46.6823 \\
\hline & \multirow{5}{*}{0.7} & 0.5 & 0.3570 & -7.1580 & 0.3415 & -6.6647 & 0.3245 & -6.1929 & 0.3057 & -5.7441 \\
\hline & & 0.6 & 0.3628 & -14.5443 & 0.3471 & -13.9698 & 0.3298 & -13.4211 & 0.3108 & -12.8998 \\
\hline & & 0.7 & 0.3695 & -23.0428 & 0.3535 & -22.3652 & 0.3360 & -21.7191 & 0.3167 & -21.1060 \\
\hline & & 0.8 & 0.3774 & -32.9307 & 0.3611 & -32.1193 & 0.3433 & -31.3472 & 0.3236 & -30.6160 \\
\hline & & 0.9 & 0.3869 & -44.5889 & 0.3701 & -43.5995 & 0.3518 & -42.6604 & 0.3318 & -41.7729 \\
\hline & \multirow{5}{*}{0.8} & 0.5 & 0.4079 & -1.0890 & 0.3965 & -0.5599 & 0.3843 & -0.0485 & 0.3711 & 0.4449 \\
\hline & & 0.6 & 0.4148 & -8.2588 & 0.4031 & -7.6385 & 0.3906 & -7.0400 & 0.3772 & -6.4636 \\
\hline & & 0.7 & 0.4229 & -16.5523 & 0.4108 & -15.8145 & 0.3980 & -15.1045 & 0.3843 & -14.4220 \\
\hline & & 0.8 & 0.4325 & -26.2659 & 0.4199 & -25.3732 & 0.4067 & -24.5167 & 0.3926 & -23.6956 \\
\hline & & 0.9 & 0.4441 & -37.8154 & 0.4310 & -36.7122 & 0.4171 & -35.6576 & 0.4025 & -34.6502 \\
\hline
\end{tabular}


Table 3.3: The PRLs in precision $L_{21}$ of the estimator $P_{2}$ with respect to $T_{1}$ at $\eta=0.25$

\begin{tabular}{|c|c|c|c|c|c|c|c|c|c|c|}
\hline \multicolumn{3}{|c|}{$\rho_{x z}$} & \multicolumn{2}{|c|}{0.5} & \multicolumn{2}{|c|}{0.6} & \multicolumn{2}{|c|}{0.7} & \multicolumn{2}{|c|}{0.8} \\
\hline$W$ & $\rho_{y x}$ & $\rho_{y z}$ & $\mu_{2}^{(0)}$ & $L_{21}$ & $\mu_{2}^{(0)}$ & $L_{21}$ & $\mu_{2}^{(0)}$ & $L_{21}$ & $\mu_{2}^{(0)}$ & $L_{21}$ \\
\hline \multirow{20}{*}{0.05} & \multirow{5}{*}{0.5} & 0.5 & 0.4466 & 1.2632 & 0.4353 & 1.2505 & 0.4219 & 1.2347 & 0.4053 & 1.2147 \\
\hline & & 0.6 & 0.4531 & 1.2703 & 0.4422 & 1.2583 & 0.4293 & 1.2434 & 0.4134 & 1.2245 \\
\hline & & 0.7 & 0.4598 & 1.2775 & 0.4493 & 1.2662 & 0.4368 & 1.2522 & 0.4216 & 1.2344 \\
\hline & & 0.8 & 0.4668 & 1.2848 & 0.4566 & 1.2741 & 0.4446 & 1.2610 & 0.4301 & 1.2443 \\
\hline & & 0.9 & 0.4742 & 1.2923 & 0.4642 & 1.2822 & 0.4527 & 1.2699 & 0.4388 & 1.2544 \\
\hline & \multirow{5}{*}{0.6} & 0.5 & 0.4792 & 1.2971 & 0.4723 & 1.2904 & 0.4647 & 1.2827 & 0.4563 & 1.2738 \\
\hline & & 0.6 & 0.4850 & 1.3026 & 0.4782 & 1.2962 & 0.4708 & 1.2888 & 0.4625 & 1.2804 \\
\hline & & 0.7 & 0.4912 & 1.3081 & 0.4845 & 1.3020 & 0.4771 & 1.2951 & 0.4689 & 1.2870 \\
\hline & & 0.8 & 0.4979 & 1.3139 & 0.4911 & 1.3080 & 0.4838 & 1.3014 & 0.4758 & 1.2938 \\
\hline & & 0.9 & 0.5052 & 1.3198 & 0.4983 & 1.3142 & 0.4910 & 1.3079 & 0.4830 & 1.3007 \\
\hline & \multirow{5}{*}{0.7} & 0.5 & 0.5023 & 1.3175 & 0.4970 & 1.3131 & 0.4914 & 1.3083 & 0.4855 & 1.3030 \\
\hline & & 0.6 & 0.5083 & 1.3222 & 0.5029 & 1.3179 & 0.4973 & 1.3133 & 0.4914 & 1.3082 \\
\hline & & 0.7 & 0.5148 & 1.3270 & 0.5093 & 1.3229 & 0.5036 & 1.3185 & 0.4976 & 1.3136 \\
\hline & & 0.8 & 0.5220 & 1.3321 & 0.5163 & 1.3281 & 0.5104 & 1.3238 & 0.5043 & 1.3191 \\
\hline & & 0.9 & 0.5301 & 1.3375 & 0.5241 & 1.3336 & 0.5180 & 1.3294 & 0.5118 & 1.3248 \\
\hline & \multirow{5}{*}{0.8} & 0.5 & 0.5216 & 1.3319 & 0.5170 & 1.3286 & 0.5122 & 1.3252 & 0.5074 & 1.3215 \\
\hline & & 0.6 & 0.5281 & 1.3362 & 0.5233 & 1.3330 & 0.5184 & 1.3297 & 0.5134 & 1.3260 \\
\hline & & 0.7 & 0.5354 & 1.3408 & 0.5304 & 1.3377 & 0.5253 & 1.3343 & 0.5201 & 1.3308 \\
\hline & & 0.8 & 0.5438 & 1.3457 & 0.5384 & 1.3426 & 0.5330 & 1.3393 & 0.5275 & 1.3358 \\
\hline & & 0.9 & 0.5534 & 1.3511 & 0.5476 & 1.3479 & 0.5418 & 1.3446 & 0.5360 & 1.3411 \\
\hline \multirow{20}{*}{0.10} & \multirow{5}{*}{0.5} & 0.5 & 0.3732 & 2.2803 & 0.3531 & 2.2325 & 0.3285 & 2.1731 & 0.2976 & 2.0972 \\
\hline & & 0.6 & 0.3846 & 2.3069 & 0.3654 & 2.2619 & 0.3420 & 2.2060 & 0.3128 & 2.1346 \\
\hline & & 0.7 & 0.3963 & 2.3336 & 0.3779 & 2.2913 & 0.3558 & 2.2389 & 0.3280 & 2.1720 \\
\hline & & 0.8 & 0.4082 & 2.3606 & 0.3907 & 2.3210 & 0.3697 & 2.2720 & 0.3435 & 2.2095 \\
\hline & & 0.9 & 0.4205 & 2.3877 & 0.4039 & 2.3508 & 0.3839 & 2.3052 & 0.3592 & 2.2472 \\
\hline & \multirow{5}{*}{0.6} & 0.5 & 0.4287 & 2.4053 & 0.4174 & 2.3809 & 0.4047 & 2.3527 & 0.3902 & 2.3196 \\
\hline & & 0.6 & 0.4381 & 2.4251 & 0.4271 & 2.4019 & 0.4148 & 2.3752 & 0.4009 & 2.3440 \\
\hline & & 0.7 & 0.4478 & 2.4450 & 0.4372 & 2.4232 & 0.4253 & 2.3980 & 0.4118 & 2.3686 \\
\hline & & 0.8 & 0.4581 & 2.4653 & 0.4477 & 2.4447 & 0.4361 & 2.4209 & 0.4231 & 2.3933 \\
\hline & & 0.9 & 0.4689 & 2.4859 & 0.4587 & 2.4665 & 0.4474 & 2.4442 & 0.4349 & 2.4183 \\
\hline & \multirow{5}{*}{0.7} & 0.5 & 0.4646 & 2.4778 & 0.4567 & 2.4626 & 0.4482 & 2.4457 & 0.4389 & 2.4267 \\
\hline & & 0.6 & 0.4733 & 2.4941 & 0.4655 & 2.4795 & 0.4571 & 2.4634 & 0.4480 & 2.4454 \\
\hline & & 0.7 & 0.4826 & 2.5106 & 0.4748 & 2.4967 & 0.4665 & 2.4814 & 0.4575 & 2.4643 \\
\hline & & 0.8 & 0.4926 & 2.5276 & 0.4847 & 2.5143 & 0.4765 & 2.4997 & 0.4676 & 2.4835 \\
\hline & & 0.9 & 0.5035 & 2.5451 & 0.4955 & 2.5324 & 0.4872 & 2.5185 & 0.4784 & 2.5031 \\
\hline & \multirow{5}{*}{0.8} & 0.5 & 0.4921 & 2.5268 & 0.4857 & 2.5160 & 0.4791 & 2.5044 & 0.4720 & 2.4917 \\
\hline & & 0.6 & 0.5009 & 2.5411 & 0.4945 & 2.5307 & 0.4878 & 2.5195 & 0.4807 & 2.5073 \\
\hline & & 0.7 & 0.5105 & 2.5559 & 0.5039 & 2.5458 & 0.4971 & 2.5350 & 0.4900 & 2.5233 \\
\hline & & 0.8 & 0.5211 & 2.5713 & 0.5143 & 2.5615 & 0.5073 & 2.5510 & 0.5001 & 2.5398 \\
\hline & & 0.9 & 0.5331 & 2.5875 & 0.5259 & 2.5779 & 0.5186 & 2.5677 & 0.5112 & 2.5569 \\
\hline
\end{tabular}




\begin{tabular}{|c|c|c|c|c|c|c|c|c|c|c|}
\hline \multicolumn{3}{|c|}{$\rho_{y z}$} & \multicolumn{2}{|c|}{0.5} & \multicolumn{2}{|c|}{0.6} & \multicolumn{2}{|c|}{0.7} & \multicolumn{2}{|c|}{0.8} \\
\hline$W$ & $\rho_{y x}$ & $\rho_{y z}$ & $\mu_{2}^{(0)}$ & $L_{21}$ & $\mu_{2}^{(0)}$ & $L_{21}$ & $\mu_{2}^{(0)}$ & $L_{21}$ & $\mu_{2}^{(0)}$ & $L_{21}$ \\
\hline \multirow{20}{*}{0.15} & \multirow{5}{*}{0.5} & 0.5 & 0.3007 & 3.0760 & 0.2718 & 2.9734 & 0.2362 & 2.8456 & 0.1913 & 2.6819 \\
\hline & & 0.6 & 0.3170 & 3.1331 & 0.2895 & 3.0365 & 0.2559 & 2.9163 & 0.2133 & 2.7624 \\
\hline & & 0.7 & 0.3335 & 3.1904 & 0.3075 & 3.0998 & 0.2756 & 2.9872 & 0.2355 & 2.8431 \\
\hline & & 0.8 & 0.3503 & 3.2479 & 0.3257 & 3.1633 & 0.2957 & 3.0582 & 0.2580 & 2.9239 \\
\hline & & 0.9 & 0.3675 & 3.3058 & 0.3442 & 3.2271 & 0.3160 & 3.1295 & 0.2806 & 3.0049 \\
\hline & \multirow{5}{*}{0.6} & 0.5 & 0.3788 & 3.3432 & 0.3632 & 3.2913 & 0.3454 & 3.2311 & 0.3248 & 3.1604 \\
\hline & & 0.6 & 0.3917 & 3.3851 & 0.3767 & 3.3360 & 0.3596 & 3.2792 & 0.3400 & 3.2126 \\
\hline & & 0.7 & 0.4050 & 3.4272 & 0.3905 & 3.3810 & 0.3741 & 3.3276 & 0.3554 & 3.2650 \\
\hline & & 0.8 & 0.4187 & 3.4698 & 0.4047 & 3.4264 & 0.3890 & 3.3763 & 0.3711 & 3.3177 \\
\hline & & 0.9 & 0.4330 & 3.5130 & 0.4195 & 3.4723 & 0.4044 & 3.4255 & 0.3873 & 3.3708 \\
\hline & \multirow{5}{*}{0.7} & 0.5 & 0.4274 & 3.4961 & 0.4168 & 3.4642 & 0.4054 & 3.4286 & 0.3928 & 3.3886 \\
\hline & & 0.6 & 0.4388 & 3.5299 & 0.4285 & 3.4996 & 0.4174 & 3.4658 & 0.4052 & 3.4279 \\
\hline & & 0.7 & 0.4508 & 3.5643 & 0.4407 & 3.5355 & 0.4298 & 3.5035 & 0.4180 & 3.4677 \\
\hline & & 0.8 & 0.4636 & 3.5992 & 0.4536 & 3.5719 & 0.4429 & 3.5417 & 0.4313 & 3.5079 \\
\hline & & 0.9 & 0.4773 & 3.6350 & 0.4673 & 3.6091 & 0.4567 & 3.5806 & 0.4454 & 3.5487 \\
\hline & \multirow{5}{*}{0.8} & 0.5 & 0.4630 & 3.5976 & 0.4548 & 3.5754 & 0.4463 & 3.5513 & 0.4371 & 3.5250 \\
\hline & & 0.6 & 0.4740 & 3.6268 & 0.4659 & 3.6055 & 0.4574 & 3.5825 & 0.4484 & 3.5574 \\
\hline & & 0.7 & 0.4859 & 3.6567 & 0.4778 & 3.6363 & 0.4693 & 3.6143 & 0.4603 & 3.5904 \\
\hline & & 0.8 & 0.4988 & 3.6875 & 0.4905 & 3.6679 & 0.4820 & 3.6469 & 0.4730 & 3.6241 \\
\hline & & 0.9 & 0.5131 & 3.7196 & 0.5046 & 3.7007 & 0.4958 & 3.6805 & 0.4867 & 3.6587 \\
\hline \multirow{20}{*}{0.20} & \multirow{5}{*}{0.5} & 0.5 & 0.2290 & 3.6723 & 0.1914 & 3.4971 & 0.1450 & 3.2786 & 0.0861 & 2.9985 \\
\hline & & 0.6 & 0.2501 & 3.7697 & 0.2145 & 3.6049 & 0.1707 & 3.3996 & 0.1150 & 3.1363 \\
\hline & & 0.7 & 0.2715 & 3.8673 & 0.2378 & 3.7129 & 0.1965 & 3.5206 & 0.1441 & 3.2743 \\
\hline & & 0.8 & 0.2931 & 3.9652 & 0.2614 & 3.8212 & 0.2225 & 3.6420 & 0.1734 & 3.4125 \\
\hline & & 0.9 & 0.3151 & 4.0636 & 0.2853 & 3.9298 & 0.2488 & 3.7636 & 0.2029 & 3.5510 \\
\hline & \multirow{5}{*}{0.6} & 0.5 & 0.3296 & 4.1271 & 0.3096 & 4.0390 & 0.2868 & 3.9366 & 0.2603 & 3.8162 \\
\hline & & 0.6 & 0.3459 & 4.1981 & 0.3268 & 4.1149 & 0.3050 & 4.0185 & 0.2798 & 3.9051 \\
\hline & & 0.7 & 0.3626 & 4.2694 & 0.3443 & 4.1912 & 0.3235 & 4.1006 & 0.2996 & 3.9943 \\
\hline & & 0.8 & 0.3798 & 4.3413 & 0.3622 & 4.2680 & 0.3424 & 4.1832 & 0.3197 & 4.0838 \\
\hline & & 0.9 & 0.3976 & 4.4139 & 0.3808 & 4.3455 & 0.3619 & 4.2664 & 0.3403 & 4.1738 \\
\hline & \multirow{5}{*}{0.7} & 0.5 & 0.3906 & 4.3856 & 0.3775 & 4.3318 & 0.3631 & 4.2716 & 0.3472 & 4.2040 \\
\hline & & 0.6 & 0.4047 & 4.4424 & 0.3920 & 4.3914 & 0.3781 & 4.3345 & 0.3629 & 4.2706 \\
\hline & & 0.7 & 0.4194 & 4.4999 & 0.4071 & 4.4517 & 0.3936 & 4.3980 & 0.3789 & 4.3377 \\
\hline & & 0.8 & 0.4349 & 4.5582 & 0.4228 & 4.5127 & 0.4097 & 4.4621 & 0.3955 & 4.4054 \\
\hline & & 0.9 & 0.4513 & 4.6177 & 0.4394 & 4.5747 & 0.4266 & 4.5271 & 0.4127 & 4.4740 \\
\hline & \multirow{5}{*}{0.8} & 0.5 & 0.4342 & 4.5556 & 0.4243 & 4.5186 & 0.4138 & 4.4783 & 0.4026 & 4.4342 \\
\hline & & 0.6 & 0.4475 & 4.6040 & 0.4377 & 4.5688 & 0.4275 & 4.5304 & 0.4165 & 4.4885 \\
\hline & & 0.7 & 0.4616 & 4.6535 & 0.4519 & 4.6198 & 0.4417 & 4.5833 & 0.4310 & 4.5436 \\
\hline & & 0.8 & 0.4767 & 4.7041 & 0.4670 & 4.6720 & 0.4569 & 4.6373 & 0.4462 & 4.5995 \\
\hline & & 0.9 & 0.4933 & 4.7564 & 0.4834 & 4.7257 & 0.4732 & 4.6926 & 0.4625 & 4.6568 \\
\hline
\end{tabular}


Table 3.4: The $P R L s$ in precision $L_{22}$ of the estimator $P_{2}$ with respect to $T_{2}$ at $\eta=0.25$

\begin{tabular}{|c|c|c|c|c|c|c|c|c|c|c|}
\hline \multicolumn{3}{|c|}{$\rho_{x z}$} & \multicolumn{2}{|c|}{0.5} & \multicolumn{2}{|c|}{0.6} & \multicolumn{2}{|c|}{0.7} & \multicolumn{2}{|c|}{0.8} \\
\hline$W$ & $\rho_{y x}$ & $\rho_{y z}$ & $\mu_{2}^{(0)}$ & $L_{22}$ & $\mu_{2}^{(0)}$ & $L_{22}$ & $\mu_{2}^{(0)}$ & $L_{22}$ & $\mu_{2}^{(0)}$ & $L_{22}$ \\
\hline \multirow{20}{*}{0.05} & \multirow{5}{*}{0.5} & 0.5 & 0.4466 & -17.7179 & 0.4353 & -17.1660 & 0.4219 & -16.6279 & 0.4053 & -16.1047 \\
\hline & & 0.6 & 0.4531 & -25.8305 & 0.4422 & -25.1944 & 0.4293 & -24.5745 & 0.4134 & -23.9719 \\
\hline & & 0.7 & 0.4598 & -35.1485 & 0.4493 & -34.4079 & 0.4368 & -33.6868 & 0.4216 & -32.9861 \\
\hline & & 0.8 & 0.4668 & -45.9632 & 0.4566 & -45.0906 & 0.4446 & -44.2421 & 0.4301 & -43.4181 \\
\hline & & 0.9 & 0.4742 & -58.6682 & 0.4642 & -57.6256 & 0.4527 & -56.6134 & 0.4388 & -55.6317 \\
\hline & \multirow{5}{*}{0.6} & 0.5 & 0.4792 & -15.2987 & 0.4723 & -14.7184 & 0.4647 & -14.1507 & 0.4563 & -13.5955 \\
\hline & & 0.6 & 0.4850 & -23.4388 & 0.4782 & -22.7672 & 0.4708 & -22.1109 & 0.4625 & -21. \\
\hline & & 0.7 & 0.4912 & -32.8239 & 0.4845 & -32.0375 & 0.4771 & -31.2704 & 0.4689 & -30.5222 \\
\hline & & 0.8 & 0.4979 & -43.7652 & 0.4911 & -42.8322 & 0.4838 & -41.9239 & 0.4758 & -41.0396 \\
\hline & & 0.9 & 0.5052 & -56.6886 & 0.4983 & -55.5638 & 0.4910 & -54.4716 & 0.4830 & -53.4107 \\
\hline & \multirow{5}{*}{0.7} & 0.5 & 0.5023 & -11.4322 & 0.4970 & -10.8328 & 0.4914 & -10.2465 & 0.4855 & -9.6729 \\
\hline & & 0.6 & 0.5083 & -19.5117 & 0.5029 & -18.8134 & 0.4973 & -18.1316 & 0.4914 & -17.4656 \\
\hline & & 0.7 & 0.5148 & -28.8686 & 0.5093 & -28.0448 & 0.5036 & -27.2420 & 0.4976 & -26.4595 \\
\hline & & 0.8 & 0.5220 & -39.8365 & 0.5163 & -38.8496 & 0.5104 & -37.8905 & 0.5043 & -36.9579 \\
\hline & & 0.9 & 0.5301 & -52.8779 & 0.5241 & -51.6740 & 0.5180 & -50.5078 & 0.5118 & -49.3772 \\
\hline & \multirow{5}{*}{0.8} & 0.5 & 0.5216 & \begin{tabular}{|l|}
-5.3787 \\
\end{tabular} & 0.5170 & -4.7715 & 0.5122 & -4.1784 & 0.5074 & -3.5986 \\
\hline & & 0.6 & 0.5281 & -13.2498 & 0.5233 & -12.5368 & 0.5184 & -11.8417 & 0.5134 & -11.1638 \\
\hline & & 0.7 & 0.5354 & -22.4149 & 0.5304 & -21.5655 & 0.5253 & -20.7396 & 0.5201 & -19.9361 \\
\hline & & 0.8 & 0.5438 & -33.2299 & 0.5384 & -32.2003 & 0.5330 & -31.2024 & 0.5275 & -30.2345 \\
\hline & & 0.9 & 0.5534 & -46.1987 & 0.5476 & -44.9234 & 0.5418 & -43.6926 & 0.5360 & -42.5034 \\
\hline \multirow{20}{*}{0.10} & \multirow{5}{*}{0.5} & 0.5 & 0.3732 & -16.5053 & 0.3531 & -16.0008 & 0.3285 & -15.5197 & 0.2976 & -15.0674 \\
\hline & & 0.6 & 0.3846 & -24.5094 & 0.3654 & -23.9219 & 0.3420 & -23.3603 & 0.3128 & -22.8297 \\
\hline & & 0.7 & 0.3963 & -33.7027 & 0.3779 & -33.0123 & 0.3558 & -32.3509 & 0.3280 & -31.7237 \\
\hline & & 0.8 & 0.4082 & -44.3726 & 0.3907 & -43.5522 & 0.3697 & -42.7652 & 0.3435 & -42.0164 \\
\hline & & 0.9 & 0.4205 & -56.9072 & 0.4039 & -55.9193 & 0.3839 & -54.9711 & 0.3592 & -54.0669 \\
\hline & \multirow{5}{*}{0.6} & 0.5 & 0.4287 & -14.0041 & 0.4174 & -13.4510 & 0.4047 & -12.9134 & 0.3902 & -12.3921 \\
\hline & & 0.6 & 0.4381 & -22.0350 & 0.4271 & -21.3918 & 0.4148 & -20.7670 & 0.4009 & -20.1610 \\
\hline & & 0.7 & 0.4478 & -31.2938 & 0.4372 & -30.5377 & 0.4253 & -29.8036 & 0.4118 & -29.0921 \\
\hline & & 0.8 & 0.4581 & -42.0878 & 0.4477 & -41.1872 & 0.4361 & -40.3141 & 0.4231 & -39.4685 \\
\hline & & 0.9 & 0.4689 & -54.8370 & 0.4587 & -53.7474 & 0.4474 & -52.6931 & 0.4349 & -51.6736 \\
\hline & \multirow{5}{*}{0.7} & 0.5 & 0.4646 & -10.1219 & 0.4567 & -9.5418 & 0.4482 & -8.9760 & 0.4389 & -8.4242 \\
\hline & & 0.6 & 0.4733 & -18.0923 & 0.4655 & -17.4149 & 0.4571 & -16.7549 & 0.4480 & -16.1122 \\
\hline & & 0.7 & 0.4826 & -27.3228 & 0.4748 & -26.5216 & 0.4665 & -25.7425 & 0.4575 & -24.9850 \\
\hline & & 0.8 & 0.4926 & -38.1422 & 0.4847 & -37.1804 & 0.4765 & -36.2473 & 0.4676 & -35.3419 \\
\hline & & 0.9 & 0.5035 & -51.0066 & 0.4955 & -49.8311 & 0.4872 & -48.6939 & 0.4784 & -47.5935 \\
\hline & \multirow{5}{*}{0.8} & 0.5 & 0.4921 & -4.1025 & 0.4857 & -3.5107 & 0.4791 & -2.9334 & 0.4720 & -2.3701 \\
\hline & & 0.6 & 0.5009 & -11.8667 & 0.4945 & -11.1708 & 0.4878 & -10.4931 & 0.4807 & -9.8330 \\
\hline & & 0.7 & 0.5105 & -20.9072 & 0.5039 & -20.0769 & 0.4971 & -19.2704 & 0.4900 & -18.4866 \\
\hline & & 0.8 & & & 0.5 & -30.5670 & 0.5073 & -29.5910 & 0.5001 & -28.6453 \\
\hline & & 0.9 & 0.5331 & -44.3663 & 0.5259 & -43.1165 & 0.5186 & -41.9111 & 0.5112 & -40.7474 \\
\hline
\end{tabular}


JAMSI, 13 (2017), No. 2

\begin{tabular}{|c|c|c|c|c|c|c|c|c|c|c|}
\hline \multicolumn{3}{|c|}{$\rho_{y z}$} & \multicolumn{2}{|c|}{0.5} & \multicolumn{2}{|c|}{0.6} & \multicolumn{2}{|c|}{0.7} & \multicolumn{2}{|c|}{0.8} \\
\hline$W$ & $\rho_{y x}$ & $\rho_{y z}$ & $\mu_{2}^{(0)}$ & $L_{22}$ & $\mu_{2}^{(0)}$ & $L_{22}$ & $\mu_{2}^{(0)}$ & $L_{22}$ & $\mu_{2}^{(0)}$ & $L_{22}$ \\
\hline \multirow{20}{*}{0.15} & \multirow{5}{*}{0.5} & 0.5 & 0.3007 & -15.5566 & 0.2718 & -15.1217 & 0.2362 & -14.7256 & 0.1913 & -14.3803 \\
\hline & & 0.6 & 0.3170 & -23.4564 & 0.2895 & -22.9398 & 0.2559 & -22.4643 & 0.2133 & -22.0417 \\
\hline & & 0.7 & 0.3335 & -32.5298 & 0.3075 & -31.9117 & 0.2756 & -31.3379 & 0.2355 & -30.8200 \\
\hline & & 0.8 & 0.3503 & -43.0605 & 0.3257 & -42.3142 & 0.2957 & -41.6166 & 0.2580 & -40.9790 \\
\hline & & 0.9 & 0.3675 & -55.4315 & 0.3442 & -54.5201 & 0.3160 & -53.6635 & 0.2806 & -52.8726 \\
\hline & \multirow{5}{*}{0.6} & 0.5 & 0.3788 & -12.9085 & 0.3632 & -12.3930 & 0.3454 & -11.8977 & 0.3248 & -11.4247 \\
\hline & & 0.6 & 0.3917 & -20.8343 & 0.3767 & -20.2300 & 0.3596 & -19.6487 & 0.3400 & -19.0923 \\
\hline & & 0.7 & 0.4050 & -29.9719 & 0.3905 & -29.2562 & 0.3741 & -28.5673 & 0.3554 & -27.9068 \\
\hline & & 0.8 & 0.4187 & -40.6244 & 0.4047 & -39.7663 & 0.3890 & -38.9403 & 0.3711 & -38.1476 \\
\hline & & 0.9 & 0.4330 & -53.2061 & 0.4195 & -52.1619 & 0.4044 & -51.1573 & 0.3873 & -50.1932 \\
\hline & \multirow{5}{*}{0.7} & 0.5 & 0.4274 & -8.9720 & 0.4168 & -8.4170 & 0.4054 & -7.8780 & 0.3928 & -7.3554 \\
\hline & & 0.6 & 0.4388 & -16.8378 & 0.4285 & -16.1867 & 0.4174 & -15.5550 & 0.4052 & -14.9427 \\
\hline & & 0.7 & 0.4508 & -25.9468 & 0.4407 & -25.1738 & 0.4298 & -24.4246 & 0.4180 & -23.6991 \\
\hline & & 0.8 & 0.4636 & -36.6234 & 0.4536 & -35.6922 & 0.4429 & -34.7913 & 0.4313 & -33.9201 \\
\hline & & 0.9 & 0.4773 & -49.3179 & 0.4673 & -48.1759 & 0.4567 & -47.0739 & 0.4454 & -46.0106 \\
\hline & \multirow{5}{*}{0.8} & 0.5 & 0.4630 & -2.9589 & 0.4548 & -2.3858 & 0.4463 & -1.8280 & 0.4371 & -1.2852 \\
\hline & & 0.6 & 0.4740 & -10.6206 & 0.4659 & -9.9448 & 0.4574 & -9.2881 & 0.4484 & -8.6500 \\
\hline & & 0.7 & 0.4859 & -19.5414 & 0.4778 & -18.7332 & 0.4693 & -17.9496 & 0.4603 & -17.1895 \\
\hline & & 0.8 & 0.4988 & -30.0674 & 0.4905 & -29.0843 & 0.4820 & -28.1337 & 0.4730 & -27.2140 \\
\hline & & 0.9 & 0.5131 & -42.6885 & 0.5046 & -41.4671 & 0.4958 & -40.2903 & 0.4867 & -39.1559 \\
\hline \multirow{20}{*}{0.20} & \multirow{5}{*}{0.5} & 0.5 & 0.2290 & -14.8457 & 0.1914 & -14.5004 & 0.1450 & -14.2143 & 0.0861 & -14.0082 \\
\hline & & 0.6 & 0.2501 & -22.6451 & 0.2145 & -22.2191 & 0.1707 & -21.8547 & 0.1150 & -21.5724 \\
\hline & & 0.7 & 0.2715 & -31.6032 & 0.2378 & -31.0772 & 0.1965 & -30.6157 & 0.1441 & -30.2394 \\
\hline & & 0.8 & 0.2931 & -41.9999 & 0.2614 & -41.3474 & 0.2225 & -40.7638 & 0.1734 & -40.2694 \\
\hline & & 0.9 & 0.3151 & -54.2133 & 0.2853 & -53.3981 & 0.2488 & -52.6577 & 0.2029 & -52.0120 \\
\hline & \multirow{5}{*}{0.6} & 0.5 & 0.3296 & -11.9928 & 0.3096 & -11.5241 & 0.2868 & -11.0819 & 0.2603 & -10.6701 \\
\hline & & 0.6 & 0.3459 & -19.8175 & 0.3268 & -19.2612 & 0.3050 & -18.7342 & 0.2798 & -18.2402 \\
\hline & & 0.7 & 0.3626 & -28.8385 & 0.3443 & -28.1724 & 0.3235 & -27.5392 & 0.2996 & -26.9426 \\
\hline & & 0.8 & 0.3798 & -39.3548 & 0.3622 & -38.5483 & 0.3424 & -37.7800 & 0.3197 & -37.0530 \\
\hline & & 0.9 & 0.3976 & -51.7756 & 0.3808 & -50.7855 & 0.3619 & -49.8410 & 0.3403 & -48.9449 \\
\hline & \multirow{5}{*}{0.7} & 0.5 & 0.3906 & -7.9676 & 0.3775 & -7.4426 & 0.3631 & -6.9362 & 0.3472 & -6.4493 \\
\hline & & 0.6 & 0.4047 & -15.7326 & 0.3920 & -15.1129 & 0.3781 & -14.5151 & 0.3629 & -13.9397 \\
\hline & & 0.7 & 0.4194 & -24.7248 & 0.4071 & -23.9849 & 0.3936 & -23.2712 & 0.3789 & -22.5843 \\
\hline & & 0.8 & 0.4349 & -35.2643 & 0.4228 & -34.3683 & 0.4097 & -33.5050 & 0.3955 & -32.6744 \\
\hline & & 0.9 & 0.4513 & -47.7953 & 0.4394 & -46.6916 & 0.4266 & -45.6301 & 0.4127 & -44.6100 \\
\hline & \multirow{5}{*}{0.8} & 0.5 & 0.4342 & -1.9358 & 0.4243 & -1.3844 & 0.4138 & -0.8494 & 0.4026 & -0.3307 \\
\hline & & 0.6 & 0.4475 & -9.4988 & 0.4377 & -8.8462 & 0.4275 & -8.2137 & 0.4165 & -7.6010 \\
\hline & & 0.7 & 0.4616 & -18.3046 & 0.4519 & -17.5214 & 0.4417 & -16.7637 & 0.4310 & -16.0309 \\
\hline & & 0.8 & 0.4767 & -28.6945 & 0.4670 & -27.7389 & 0.4569 & -26.8166 & 0.4462 & -25.9264 \\
\hline & & 0.9 & 0.4933 & -41.1519 & 0.4834 & -39.9613 & 0.4732 & -38.8162 & 0.4625 & -37.7143 \\
\hline
\end{tabular}


Table 3.5: The $P R L s$ in precision $L_{1}$ of the estimator $P_{1}$ with respect to $\bar{y}_{n}^{*}$ at $\eta=0.25$

\begin{tabular}{|c|c|c|c|c|c|c|c|c|c|c|}
\hline \multicolumn{3}{|c|}{$\rho_{x z}$} & \multicolumn{2}{|c|}{0.5} & \multicolumn{2}{|c|}{0.6} & \multicolumn{2}{|c|}{0.7} & \multicolumn{2}{|c|}{0.8} \\
\hline$W$ & $\rho_{y x}$ & $\rho_{y z}$ & $\mu_{1}^{(0)}$ & $L_{1}$ & $\mu_{1}^{(0)}$ & $L_{1}$ & $\mu_{1}^{(0)}$ & $L_{1}$ & $\mu_{1}^{(0)}$ & $L_{1}$ \\
\hline \multirow{20}{*}{0.05} & \multirow{5}{*}{0.5} & 0.5 & 0.4296 & 30.3788 & 0.4163 & -29.7757 & 0.4002 & -29.1899 & 0.3803 & -28.6233 \\
\hline & & 0.6 & 0.4317 & -39.2348 & 0.4182 & -38.5425 & 0.4020 & -37.8708 & 0.3819 & -37.2220 \\
\hline & & 0.7 & 0.4341 & -49.3862 & 0.4204 & -48.5832 & 0.4040 & -47.8053 & 0.3837 & -47.0550 \\
\hline & & 0.8 & 0.4369 & -61.1410 & 0.4229 & -60.1984 & 0.4063 & -59.2869 & 0.3858 & -58.4092 \\
\hline & & 0.9 & 0.4402 & -74.9138 & 0.4260 & -73.7916 & 0.4091 & -72.7087 & 0.3883 & -71.6682 \\
\hline & \multirow{5}{*}{0.6} & 0.5 & 0.4675 & -32.9470 & 0.4596 & -32.2822 & 0.4508 & -31.6325 & 0.4409 & -30.9979 \\
\hline & & 0.6 & 0.4704 & \begin{tabular}{|l|}
-42.1939 \\
\end{tabular} & 0.4622 & -41.4262 & 0.4533 & -40.6771 & 0.4432 & -39.9466 \\
\hline & & 0.7 & 0.4737 & -52.8332 & 0.4653 & -51.9365 & 0.4561 & -51.0632 & 0.4458 & -50.2131 \\
\hline & & 0.8 & 0.4776 & -65.2080 & 0.4689 & -64.1467 & 0.4594 & -63.1154 & 0.4489 & -62.1135 \\
\hline & & 0.9 & 0.4822 & -79.7857 & 0.4732 & -78.5095 & 0.4634 & -77.2727 & 0.4525 & -76.0743 \\
\hline & \multirow{5}{*}{0.7} & 0.5 & 0.4936 & -35.7614 & 0.4877 & -35.0339 & 0.4814 & -34.3225 & 0.4747 & -33.6270 \\
\hline & & 0.6 & 0.4973 & -45.4580 & 0.4912 & -44.6120 & 0.4847 & -43.7863 & 0.4778 & -42.9805 \\
\hline & & 0.7 & 0.5017 & \begin{tabular}{|l|}
-56.6651 \\
\end{tabular} & 0.4953 & -55.6687 & 0.4885 & -54.6986 & 0.4814 & -53.7537 \\
\hline & & 0.8 & 0.5070 & \begin{tabular}{|l|}
-69.7714 \\
\end{tabular} & 0.5002 & -68.5804 & 0.4931 & -67.4237 & 0.4856 & -66.3000 \\
\hline & & 0.9 & 0.5132 & -85.3148 & .5060 & -83.8649 & 0.4985 & -82.4616 & 0.4906 & -81.1025 \\
\hline & \multirow{5}{*}{0.8} & 0.5 & 0.5148 & -38.8448 & 0.5097 & -38.0468 & 0.5045 & -37.2673 & 0.4992 & -36.5057 \\
\hline & & 0.6 & 0.5196 & -49.0618 & 0.5143 & -48.1261 & 0.5088 & -47.2142 & 0.5032 & -46.3252 \\
\hline & & 0.7 & 0.5254 & -60.9349 & 0.5197 & -59.8222 & 0.5139 & -58.7405 & 0.5079 & -57.6886 \\
\hline & & 0.8 & 0.5322 & -74.9141 & 0.5261 & -73.5676 & 0.5199 & -72.2631 & 0.5135 & -70.9984 \\
\hline & & 0.9 & 0.5405 & -91.6344 & 0.5339 & -89.9701 & 0.5271 & -88.3644 & 0.5203 & -86.8136 \\
\hline \multirow{20}{*}{0.10} & \multirow{5}{*}{0.5} & 0.5 & 0.3396 & -32.3323 & 0.3154 & -31.7906 & 0.2858 & -31.2830 & 0.2484 & -30 \\
\hline & & 0.6 & 0.3424 & -41.2 & 3180 & -40.5909 & 0.2882 & -40.0081 & 0.2507 & -39.4752 \\
\hline & & 0.7 & 0.3456 & -51.3776 & 0.3210 & -50.6549 & 0.2910 & -49.9789 & 0.2533 & -49.3612 \\
\hline & & 0.8 & 0.3492 & -63.1264 & 0.3245 & -62.2771 & 0.2943 & -61.4837 & 0.2564 & -60.7593 \\
\hline & & 0.9 & 0.3536 & -76.8641 & 0.3285 & -75.8518 & 0.2981 & -74.9073 & 0.2599 & $\begin{array}{l}-74.0460 \\
\end{array}$ \\
\hline & \multirow{5}{*}{0.6} & 0.5 & 0.4056 & -34.7515 & 0.3923 & -34.1138 & 0.3772 & -33.4969 & 0.3599 & -32.9023 \\
\hline & & 0.6 & 0.4091 & -44.0015 & 0.3956 & -43.2652 & 0.3803 & -42.5538 & 0.3628 & -41.8690 \\
\hline & & 0.7 & 0.4132 & -54.6265 & 0.3994 & -53.7664 & 0.3839 & -52.9370 & 0.3662 & -52.1397 \\
\hline & & 0.8 & 0.4179 & -66.9610 & 0.4039 & -65.9431 & 0.3881 & -64.9633 & 0.3701 & -64.0234 \\
\hline & & 0.9 & 0.4236 & -81.4594 & 0.4092 & -80.2354 & 0.3931 & -79.0602 & 0.3748 & -77.9354 \\
\hline & \multirow{5}{*}{0.7} & 0.5 & 0.4474 & -37.4950 & 0.4382 & -36.7808 & 0.4284 & -36.0854 & 0.4176 & -35.4089 \\
\hline & & 0.6 & 0.4518 & -47.1827 & 0.4424 & -46.3525 & 0.4323 & -45.5455 & 0.4213 & -44.7618 \\
\hline & & 0.7 & 0.4569 & -58.3601 & 0.4473 & -57.3827 & 0.4369 & -56.4348 & 0.4256 & -55.5161 \\
\hline & & 0.8 & 0.4630 & -71.4059 & 0.4530 & -70.2382 & 0.4423 & -69.1085 & 0.4306 & -68.0162 \\
\hline & & 0.9 & 0.4703 & -86.8429 & 0.4599 & -85.4222 & 0.4487 & -84.0522 & 0.4367 & -82.7315 \\
\hline & \multirow{5}{*}{0.8} & 0.5 & 0.4786 & -40.5443 & 0.4714 & -39.7524 & 0.4639 & -38.9805 & 0.4559 & -38.2282 \\
\hline & & 0.6 & 0.4841 & -50.7449 & 0.4767 & -49.8170 & 0.4688 & -48.9144 & 0.4606 & -48.0366 \\
\hline & & 0.7 & 0.4906 & -62.5783 & 0.4828 & -61.4754 & 0.4746 & -60.4055 & 0.4660 & -59.3674 \\
\hline & & 0.8 & 0.4984 & & 0.4901 & -75.1497 & 0.4815 & -73.8602 & 0.4725 & -72.6129 \\
\hline & & 0.9 & 0.5078 & \begin{tabular}{|l|}
-93.0775 \\
\end{tabular} & 0.4989 & -91.4306 & 0.4898 & -89.8446 & 0.4803 & -88.3163 \\
\hline
\end{tabular}




\begin{tabular}{|c|c|c|c|c|c|c|c|c|c|c|}
\hline \multicolumn{3}{|c|}{$\rho_{x z}$} & \multicolumn{2}{|c|}{0.5} & \multicolumn{2}{|c|}{0.6} & \multicolumn{2}{|c|}{0.7} & \multicolumn{2}{|c|}{0.8} \\
\hline$W$ & $\rho_{y x}$ & $\rho_{y z}$ & $\mu_{1}^{(0)}$ & $L_{1}$ & $\mu_{1}^{(0)}$ & $L_{1}$ & $\mu_{1}^{(0)}$ & $L_{1}$ & $\mu_{1}^{(0)}$ & $L_{1}$ \\
\hline \multirow{20}{*}{0.15} & \multirow{5}{*}{0.5} & 0.5 & 0.2510 & -34.6492 & 0.2161 & -34.2105 & 0.1730 & -33.8331 & 0.1183 & -33.5379 \\
\hline & & 0.6 & 0.2545 & -43.6041 & 0.2194 & -43.0980 & 0.1762 & -42.6621 & 0.1215 & -42.3198 \\
\hline & & 0.7 & 0.2585 & -53.8407 & 0.2232 & -53.2504 & 0.1799 & -52.7414 & 0.1250 & -52.3402 \\
\hline & & 0.8 & 0.2631 & -65.6571 & 0.2277 & -64.9599 & 0.1842 & -64.3580 & 0.1291 & -63.8816 \\
\hline & & 0.9 & 0.2686 & -79.4521 & 0.2329 & -78.6164 & 0.1891 & -77.8940 & 0.1339 & -77.3195 \\
\hline & \multirow{5}{*}{0.6} & 0.5 & 0.3447 & -36.8126 & 0.3260 & -36.2226 & 0.3047 & -35.6625 & 0.2801 & -35.1361 \\
\hline & & 0.6 & 0.3489 & -46.1024 & 0.3300 & -45.4203 & 0.3085 & -44.7733 & 0.2837 & -44.1657 \\
\hline & & 0.7 & 0.3537 & -56.7583 & 0.3346 & -55.9605 & 0.3129 & -55.2047 & 0.2879 & -54.4957 \\
\hline & & 0.8 & 0.3594 & -69.1091 & 0.3400 & -68.1634 & 0.3181 & -67.2688 & 0.2928 & -66.4307 \\
\hline & & 0.9 & 0.3661 & -83.6004 & 0.3464 & -82.4612 & 0.3241 & -81.3858 & 0.2985 & -80.3798 \\
\hline & \multirow{5}{*}{0.7} & 0.5 & 0.4018 & -39.4260 & 0.3895 & -38.7373 & 0.3761 & -38.0715 & 0.3612 & -37.4295 \\
\hline & & 0.6 & 0.4069 & -49.1349 & 0.3944 & -48.3340 & 0.3807 & -47.5610 & 0.3656 & -46.8167 \\
\hline & & 0.7 & 0.4129 & -60.3200 & 0.4000 & -59.3768 & 0.3860 & -58.4682 & 0.3707 & -57.5949 \\
\hline & & 0.8 & 0.4199 & -73.3530 & 0.4067 & -72.2254 & 0.3923 & -71.1418 & 0.3767 & -70.1027 \\
\hline & & 0.9 & 0.4282 & -88.7450 & 0.4146 & -87.3725 & 0.3998 & -86.0574 & 0.3838 & -84.7998 \\
\hline & \multirow{5}{*}{0.8} & 0.5 & 0.4430 & -42.4035 & 0.4337 & -41.6258 & 0.4238 & -40.8704 & 0.4132 & -40.1372 \\
\hline & & 0.6 & 0.4492 & -52.6137 & 0.4396 & -51.7024 & 0.4294 & -50.8191 & 0.4186 & -49.9633 \\
\hline & & 0.7 & 0.4565 & -64.4401 & 0.4465 & -63.3570 & 0.4360 & -62.3098 & 0.4248 & -61.2976 \\
\hline & & 0.8 & 0.4651 & -78.3128 & 0.4547 & -77.0033 & 0.4438 & -75.7411 & 0.4322 & -74.5247 \\
\hline & & 0.9 & 0.4757 & -94.8362 & 0.4646 & -93.2191 & 0.4531 & -91.6666 & 0.4410 & -90.1759 \\
\hline \multirow{20}{*}{0.20} & \multirow{5}{*}{0.5} & 0.5 & 0.1636 & -37.3094 & 0.1181 & -37.0133 & 0.0618 & -36.8157 & $*$ & - \\
\hline & & 0.6 & 0.1679 & -46.3812 & 0.1222 & -46.0351 & 0.0659 & -45.8009 & $*$ & - \\
\hline & & 0.7 & 0.1728 & -56.7418 & 0.1270 & -56.3325 & 0.0705 & -56.0515 & $*$ & - \\
\hline & & 0.8 & 0.1785 & -68.6886 & 0.1325 & -68.1979 & 0.0759 & -67.8557 & 0.0040 & -67.7152 \\
\hline & & 0.9 & 0.1851 & -82.6187 & 0.1390 & -82.0207 & 0.0822 & -81.5970 & 0.0102 & -81.4085 \\
\hline & \multirow{5}{*}{0.6} & 0.5 & 0.2845 & -39.1156 & 0.2606 & -38.5928 & 0.2332 & -38.1121 & 0.2013 & -37.6809 \\
\hline & & 0.6 & 0.2895 & -48.4775 & 0.2654 & -47.8711 & 0.2378 & -47.3136 & 0.2058 & -46.8131 \\
\hline & & 0.7 & 0.2952 & -59.2035 & 0.2708 & -58.4918 & 0.2431 & -57.8376 & 0.2108 & -57.2499 \\
\hline & & 0.8 & 0.3018 & -71.6191 & 0.2772 & -70.7721 & 0.2492 & -69.9939 & 0.2167 & -69.2945 \\
\hline & & 0.9 & 0.3096 & -86.1640 & 0.2847 & -85.1394 & 0.2563 & -84.1987 & 0.2236 & -83.3529 \\
\hline & \multirow{5}{*}{0.7} & 0.5 & 0.3570 & -41.5428 & 0.3415 & -40.8912 & 0.3245 & -40.2680 & 0.3057 & -39.6752 \\
\hline & & 0.6 & 0.3628 & -51.2993 & 0.3471 & -50.5404 & 0.3298 & -49.8157 & 0.3108 & -49.1271 \\
\hline & & 0.7 & 0.3695 & -62.5246 & 0.3535 & -61.6296 & 0.3360 & -60.7762 & 0.3167 & -59.9665 \\
\hline & & 0.8 & 0.3774 & -75.5854 & 0.3611 & -74.5137 & 0.3433 & -73.4939 & 0.3236 & -72.5279 \\
\hline & & 0.9 & 0.3869 & -90.9845 & 0.3701 & -89.6776 & 0.3518 & -88.4372 & 0.3318 & -87.2649 \\
\hline & \multirow{5}{*}{0.8} & 0.5 & 0.4079 & -44.4128 & 0.3965 & -43.6570 & 0.3843 & -42.9265 & 0.3711 & -42.2216 \\
\hline & & 0.6 & 0.4148 & -54.6554 & 0.4031 & -53.7692 & 0.3906 & -52.9143 & 0.3772 & -52.0909 \\
\hline & & 0.7 & 0.4229 & -66.5032 & 0.4108 & -65.4493 & 0.3980 & -64.4349 & 0.3843 & -63.4600 \\
\hline & & 0.8 & 0.4325 & -80.3798 & 0.4199 & -79.1046 & 0.4067 & -77.8809 & 0.3926 & -76.7079 \\
\hline & & 0.9 & 0.4441 & -96.8791 & 0.4310 & -95.3032 & 0.4171 & -93.7965 & 0.4025 & -92.3574 \\
\hline
\end{tabular}


Table 3.6: The PRLs in precision $L_{2}$ of the estimator $P_{2}$ with respect to $\bar{y}_{n}^{*}$ at $\eta=0.25$

\begin{tabular}{|c|c|c|c|c|c|c|c|c|c|c|}
\hline \multicolumn{3}{|l|}{$\rho_{x z}$} & \multicolumn{2}{|l|}{0.5} & \multicolumn{2}{|l|}{0.6} & \multicolumn{2}{|l|}{0.7} & \multicolumn{2}{|l|}{0.8} \\
\hline$W$ & $\rho_{y x}$ & $\rho_{y z}$ & $\mu_{2}^{(0)}$ & $L_{2}$ & $\mu_{2}^{(0)}$ & $L_{2}$ & $\mu_{2}^{(0)}$ & $L_{2}$ & $\mu_{2}^{(0)}$ & $L_{2}$ \\
\hline \multirow{20}{*}{0.05} & \multirow{5}{*}{0.5} & 0.5 & 0.4466 & -30.7172 & 0.4353 & -30.1043 & 0.4219 & -29.5068 & 0.4053 & -28.9258 \\
\hline & & 0.6 & 0.4531 & -39.7256 & 0.4422 & -39.0193 & 0.4293 & -38.3310 & 0.4134 & -37.6618 \\
\hline & & 0.7 & 0.4598 & -50.0726 & 0.4493 & -49.2502 & 0.4368 & -48.4495 & 0.4216 & -47.6714 \\
\hline & & 0.8 & 0.4668 & -62.0815 & 0.4566 & -61.1126 & 0.4446 & -60.1703 & 0.4301 & -59.2554 \\
\hline & & 0.9 & 0.4742 & -76.1895 & 0.4642 & -75.0318 & 0.4527 & \begin{tabular}{|l|}
-73.9078 \\
\end{tabular} & 0.4388 & -72.8176 \\
\hline & \multirow{5}{*}{0.6} & 0.5 & 0.4792 & -33.3141 & 0.4723 & -32.6432 & 0.4647 & -31.9868 & 0.4563 & -31.3448 \\
\hline & & 0.6 & 0.4850 & -42.7262 & 0.4782 & -41.9495 & 0.4708 & -41.1907 & 0.4625 & -40.4495 \\
\hline & & 0.7 & 0.4912 & -53.5776 & 0.4845 & -52.6684 & 0.4771 & -51.7814 & 0.4689 & -50.9163 \\
\hline & & 0.8 & 0.4979 & -66.2285 & 0.4911 & -65.1497 & 0.4838 & -64.0995 & 0.4758 & -63.0770 \\
\hline & & 0.9 & 0.5052 & \begin{tabular}{|l|}
-81.1712 \\
\end{tabular} & 0.4983 & -79.8706 & 0.4910 & -78.6078 & .4830 & -77. \\
\hline & \multirow{5}{*}{0.7} & 0.5 & 0.5023 & -36.1493 & 0.4970 & -35.4169 & 0.4914 & -34.7006 & 0.4855 & -33.9998 \\
\hline & & 0.6 & 0.5083 & -46.0210 & 0.5029 & -45.1678 & 0.4973 & -44.3347 & 0.4914 & -43.5211 \\
\hline & & 0.7 & \begin{tabular}{|l|}
0.5148 \\
\end{tabular} & \begin{tabular}{|l|}
-57.4534 \\
\end{tabular} & 0.5093 & \begin{tabular}{|l|}
-56.4468 \\
\end{tabular} & 0.5036 & \begin{tabular}{|l|}
-55.4660 \\
\end{tabular} & \begin{tabular}{|l|}
0.4976 \\
\end{tabular} & -54.5099 \\
\hline & & 0.8 & 0.5220 & -70.8541 & 0.5163 & -69.6483 & 0.5104 & -68.4765 & 0.5043 & -67.3370 \\
\hline & & 0.9 & 0.5301 & -86.7883 & 0.5241 & -85.3174 & 0.5180 & -83.8924 & 0.5118 & -82.5 \\
\hline & \multirow{5}{*}{0.8} & 0.5 & 0.5216 & -39.2505 & 0.5170 & -38.4481 & 0.5122 & -37.6643 & \begin{tabular}{|l|}
0.5074 \\
\end{tabular} & -36. \\
\hline & & 0.6 & 0.5281 & -49.6515 & 0.5233 & -48.7093 & 0.5184 & -47.7908 & 0.5134 & -46.8950 \\
\hline & & 0.7 & 0.5354 & -61.7625 & 0.5304 & -60.6401 & 0.5253 & -59.5488 & 0.5201 & -58.4870 \\
\hline & & 0.8 & 0.5438 & -76.0538 & 0.5384 & -74.6932 & 0.5330 & \begin{tabular}{|l|}
-73.3745 \\
\end{tabular} & 0.5275 & -72.0955 \\
\hline & & 0.9 & 0.5534 & -93.1911 & 0.5476 & -91.5060 & 0.5418 & -89.8795 & 0.5360 & -88.3080 \\
\hline \multirow{20}{*}{0.10} & \multirow{5}{*}{0.5} & 0.5 & 0.3732 & -32.8672 & 0.3531 & -32.2918 & 0.3285 & -31.7432 & 0.2976 & -31.2273 \\
\hline & & 0.6 & 0.3846 & -41.9953 & 0.3654 & -41.3254 & 0.3420 & -40.6849 & 0.3128 & -40.0798 \\
\hline & & 0.7 & 0.3963 & -52.4798 & 0.3779 & -51.6924 & 0.3558 & -50.9381 & 0.3280 & -50.2228 \\
\hline & & 0.8 & 0.4082 & -64.6481 & 0.3907 & -63.7124 & 0.3697 & -62.8149 & 0.3435 & -61.9611 \\
\hline & & 0.9 & 0.4205 & -78.9431 & 0.4039 & -77.8165 & 0.3839 & -76.7350 & 0.3592 & -75.7039 \\
\hline & \multirow{5}{*}{0.6} & 0.5 & 0.4287 & -35.3799 & 0.4174 & -34.7231 & 0.4047 & -34.0847 & 0.3902 & -33.4656 \\
\hline & & 0.6 & 0.4381 & -44.9165 & 0.4271 & -44.1528 & 0.4148 & -43.4108 & 0.4009 & -42.6912 \\
\hline & & 0.7 & 0.4478 & -55.9114 & 0.4372 & -55.0135 & 0.4253 & -54.1418 & 0.4118 & -53.2969 \\
\hline & & 0.8 & 0.4581 & -68.7293 & 0.4477 & -67.6598 & 0.4361 & -66.6229 & 0.4231 & -65.6188 \\
\hline & & 0.9 & 0.4689 & -83.8689 & 0.4587 & \begin{tabular}{|l|}
-82.5751 \\
\end{tabular} & 0.4474 & -81.3231 & 0.4349 & -80.1124 \\
\hline & \multirow{5}{*}{0.7} & 0.5 & 0.4646 & -38.1848 & 0.4567 & -37.4569 & 0.4482 & -36.7469 & 0.4389 & -36.0545 \\
\hline & & 0.6 & 0.4733 & -48.1865 & 0.4655 & -47.3363 & 0.4571 & -46.5082 & 0.4480 & -45.7017 \\
\hline & & 0.7 & 0.4826 & \begin{tabular}{|l|}
-59.7692 \\
\end{tabular} & 0.4748 & \begin{tabular}{|l|}
-58.7638 \\
\end{tabular} & 0.4665 & -57.7862 & 0.4575 & -56.8355 \\
\hline & & 0.8 & 0.4926 & -73.3457 & 0.4847 & -72.1388 & 0.4765 & -70.9679 & 0.4676 & -69.8318 \\
\hline & & 0.9 & 0.5035 & -89.4885 & 0.4955 & -88.0133 & 0.4872 & -86.5864 & 0.4784 & -85.2055 \\
\hline & \multirow{5}{*}{0.8} & 0.5 & 0.4921 & -41.2820 & 0.4857 & -40.4789 & 0.4791 & -39.6953 & 0.4720 & -38.9308 \\
\hline & & 0.6 & 0.5009 & -51.8192 & 0.4945 & -50.8746 & 0.4878 & -49.9549 & 0.4807 & -49.0591 \\
\hline & & 0.7 & 0.5105 & -64.0884 & 0.5039 & -62.9615 & 0.4971 & -61.8669 & 0.4900 & -60.8032 \\
\hline & & 0.8 & 0.5211 & \begin{tabular}{|l|}
-78.5658 \\
\end{tabular} & 0.5143 & \begin{tabular}{|l|}
-77.1980 \\
\end{tabular} & 0.5073 & -75.8735 & 0.5001 & -74.5900 \\
\hline & & 0.9 & 0.5331 & -95.9256 & 0.5259 & -94.2295 & 0.5186 & -92.5936 & 0.5112 & -91.0143 \\
\hline
\end{tabular}


JAMSI, 13 (2017), No. 2

\begin{tabular}{|c|c|c|c|c|c|c|c|c|c|c|}
\hline \multicolumn{3}{|c|}{$\rho_{y z}$} & \multicolumn{2}{|c|}{0.5} & \multicolumn{2}{|c|}{0.6} & \multicolumn{2}{|c|}{0.7} & \multicolumn{2}{|c|}{0.8} \\
\hline$W$ & $\rho_{y x}$ & $\rho_{y z}$ & $\mu_{2}^{(0)}$ & $L_{2}$ & $\mu_{2}^{(0)}$ & $L_{2}$ & $\mu_{2}^{(0)}$ & $L_{2}$ & $\mu_{2}^{(0)}$ & $L_{2}$ \\
\hline \multirow{20}{*}{0.15} & \multirow{5}{*}{0.5} & 0.5 & 0.3007 & -35.2533 & 0.2718 & -34.7443 & 0.2362 & -34.2807 & 0.1913 & -33.8764 \\
\hline & & 0.6 & 0.3170 & -44.4995 & 0.2895 & -43.8949 & 0.2559 & -43.3383 & 0.2133 & -42.8438 \\
\hline & & 0.7 & 0.3335 & -55.1196 & 0.3075 & -54.3961 & 0.2756 & -53.7245 & 0.2355 & -53.1184 \\
\hline & & 0.8 & 0.3503 & -67.4452 & 0.3257 & -66.5717 & 0.2957 & -65.7552 & 0.2580 & -65.0089 \\
\hline & & 0.9 & 0.3675 & -81.9248 & 0.3442 & -80.8581 & 0.3160 & -79.8555 & 0.2806 & -78.9298 \\
\hline & \multirow{5}{*}{0.6} & 0.5 & 0.3788 & -37.6072 & 0.3632 & -36.9790 & 0.3454 & -36.3753 & 0.3248 & -35.7988 \\
\hline & & 0.6 & 0.3917 & -47.2668 & 0.3767 & -46.5303 & 0.3596 & -45.8218 & 0.3400 & -45.1437 \\
\hline & & 0.7 & 0.4050 & -58.4033 & 0.3905 & -57.5310 & 0.3741 & -56.6914 & 0.3554 & -55.8865 \\
\hline & & 0.8 & 0.4187 & -71.3860 & 0.4047 & -70.3402 & 0.3890 & -69.3335 & 0.3711 & -68.3674 \\
\hline & & 0.9 & 0.4330 & -86.7200 & 0.4195 & -85.4473 & 0.4044 & -84.2229 & 0.3873 & -83.0479 \\
\hline & \multirow{5}{*}{0.7} & 0.5 & 0.4274 & -40.3404 & 0.4168 & -39.6256 & 0.4054 & -38.9315 & 0.3928 & -38.2585 \\
\hline & & 0.6 & 0.4388 & -50.4704 & 0.4285 & -49.6319 & 0.4174 & -48.8183 & 0.4052 & -48.0298 \\
\hline & & 0.7 & 0.4508 & -62.2015 & 0.4407 & -61.2059 & 0.4298 & -60.2411 & 0.4180 & -59.3069 \\
\hline & & 0.8 & 0.4636 & -75.9515 & 0.4536 & -74.7522 & 0.4429 & -73.5919 & 0.4313 & -72.4700 \\
\hline & & 0.9 & 0.4773 & -92.3001 & 0.4673 & -90.8295 & 0.4567 & -89.4102 & 0.4454 & -88.0408 \\
\hline & \multirow{5}{*}{0.8} & 0.5 & 0.4630 & -43.4070 & 0.4548 & -42.6089 & 0.4463 & -41.8319 & 0.4371 & -41.0758 \\
\hline & & 0.6 & 0.4740 & -54.0786 & 0.4659 & -53.1374 & 0.4574 & -52.2227 & 0.4484 & -51.3339 \\
\hline & & 0.7 & 0.4859 & -66.5041 & 0.4778 & -65.3784 & 0.4693 & -64.2869 & 0.4603 & -63.2282 \\
\hline & & 0.8 & 0.4988 & -81.1653 & 0.4905 & -79.7960 & 0.4820 & -78.4719 & 0.4730 & -77.1909 \\
\hline & & 0.9 & 0.5131 & -98.7447 & 0.5046 & -97.0434 & 0.4958 & -95.4044 & 0.4867 & -93.8243 \\
\hline \multirow{20}{*}{0.20} & \multirow{5}{*}{0.5} & 0.5 & 0.2290 & -37.8678 & 0.1914 & -37.4534 & 0.1450 & -37.1099 & 0.0861 & -36.8625 \\
\hline & & 0.6 & 0.2501 & -47.2307 & 0.2145 & -46.7194 & 0.1707 & -46.2819 & 0.1150 & -45.9431 \\
\hline & & 0.7 & 0.2715 & -57.9846 & 0.2378 & -57.3531 & 0.1965 & -56.7992 & 0.1441 & -56.3474 \\
\hline & & 0.8 & 0.2931 & -70.4654 & 0.2614 & -69.6822 & 0.2225 & -68.9816 & 0.1734 & -68.3880 \\
\hline & & 0.9 & 0.3151 & -85.1272 & 0.2853 & -84.1485 & 0.2488 & -83.2597 & 0.2029 & -82.4846 \\
\hline & \multirow{5}{*}{0.6} & 0.5 & 0.3296 & -39.9910 & 0.3096 & -39.4051 & 0.2868 & -38.8523 & 0.2603 & -38.3376 \\
\hline & & 0.6 & 0.3459 & -49.7719 & 0.3268 & -49.0765 & 0.3050 & -48.4178 & 0.2798 & -47.8002 \\
\hline & & 0.7 & 0.3626 & -61.0481 & 0.3443 & -60.2155 & 0.3235 & -59.4240 & 0.2996 & -58.6782 \\
\hline & & 0.8 & 0.3798 & -74.1936 & 0.3622 & -73.1854 & 0.3424 & -72.2249 & 0.3197 & -71.3162 \\
\hline & & 0.9 & 0.3976 & -89.7194 & 0.3808 & -88.4818 & 0.3619 & -87.3013 & 0.3403 & -86.1812 \\
\hline & \multirow{5}{*}{0.7} & 0.5 & 0.3906 & -42.6122 & 0.3775 & -41.9187 & 0.3631 & -41.2498 & 0.3472 & -40.6067 \\
\hline & & 0.6 & 0.4047 & -52.8688 & 0.3920 & -52.0503 & 0.3781 & -51.2606 & 0.3629 & -50.5006 \\
\hline & & 0.7 & 0.4194 & -64.7464 & 0.4071 & -63.7690 & 0.3936 & -62.8264 & 0.3789 & -61.9191 \\
\hline & & 0.8 & 0.4349 & -78.6678 & 0.4228 & -77.4843 & 0.4097 & -76.3441 & 0.3955 & -75.2469 \\
\hline & & 0.9 & 0.4513 & -95.2197 & 0.4394 & -93.7619 & 0.4266 & -92.3597 & 0.4127 & -91.0123 \\
\hline & \multirow{5}{*}{0.8} & 0.5 & 0.4342 & -45.6225 & 0.4243 & -44.8349 & 0.4138 & -44.0706 & 0.4026 & -43.3296 \\
\hline & & 0.6 & 0.4475 & -56.4269 & 0.4377 & -55.4946 & 0.4275 & -54.5910 & 0.4165 & -53.7158 \\
\hline & & 0.7 & 0.4616 & -69.0065 & 0.4519 & -67.8877 & 0.4417 & -66.8053 & 0.4310 & -65.7584 \\
\hline & & 0.8 & 0.4767 & -83.8493 & 0.4670 & -82.4841 & 0.4569 & -81.1665 & 0.4462 & $\begin{array}{l}-79.8949 \\
\end{array}$ \\
\hline & & 0.9 & 0.4933 & -101.6456 & 0.4834 & -99.9448 & 0.4732 & -98.3088 & 0.4625 & -96.7347 \\
\hline
\end{tabular}


It is observed from Table 3.1, that:

(i) for fixed values of $\left(W, f_{2}, \eta=0.25, \rho_{\mathrm{yx}}, \rho_{\mathrm{xz}} \rho_{\mathrm{xz}}\right)$, the values of $\mu_{1}^{(0)}$ and $L_{11}$ increase as the value of $\rho_{\mathrm{yz}}$ increases. The efficiency of an estimator utilizing auxiliary variable under complete response will increase with increasing value of the correlation coefficients between the study variable and the auxiliary variable on which the estimator is developed. Similar trend is observed for fixed values of ( $W$, $\left.f_{2}, \eta=0.25, \eta=0.25, \rho_{\mathrm{yz}}, \rho_{\mathrm{xz}}\right)$ with increasing values of $\rho_{\mathrm{yx}}$.

(ii) for fixed values of $\left(W, f_{2}, \eta=0.25, \rho_{\mathrm{yz}}, \rho_{\mathrm{yx}}\right)$, the values of $\mu_{1}^{(0)}$ and $L_{11}$ decrease with increasing values of $\rho_{\mathrm{xz}}$.

(iii) for fixed values of $\left(f_{2}, \eta=0.25, \rho_{\mathrm{yx}}, \rho_{\mathrm{yz}}, \rho_{\mathrm{xz}}\right)$, the values of $\mu_{1}^{(0)}$ decrease while the value of $L_{11}$ increases with increasing values of $W$. This pattern demonstrates that with the higher non-response rate one may require selecting the smaller sample on the current occasion, which reduces the cost of the survey.

From Table 3.2, it is visible that:

(i) for fixed values of $\left(W, f_{2}, \eta=0.25, \rho_{\mathrm{yx}}, \rho_{\mathrm{xz}}\right)$, the values of $\mu_{1}^{(0)}$ increase while the values of $L_{12}$ decrease with the increasing values of $\rho_{\mathrm{yz}}$. This implies that if one uses the information on highly correlated auxiliary variable, there is a significance gain in the precision of estimate.

(ii) for fixed values of $\left(W, f_{2}, \eta=0.25, \rho_{\mathrm{yx}}, \rho_{\mathrm{yz}}\right)$, the values of $\mu_{1}^{(0)}$ decrease while the values of $L_{12}$ increases with the increasing values of $\rho_{\mathrm{xz}}$.

(iii) for fixed values of $\left(W, f_{2}, \eta=0.25, \rho_{\mathrm{yz}}, \rho_{\mathrm{xz}}\right)$, the values of $\mu_{1}^{(0)}$ and $L_{12}$ increase with increasing values of $\rho_{\mathrm{yx}}$.

(iv) for fixed values of $\left(f_{2}, \eta=0.25, \rho_{\mathrm{yx}}, \rho_{\mathrm{yz}}, \rho_{\mathrm{xz}}\right)$, the values of $\mu_{1}^{(0)}$ decrease while the value of $L_{12}$ increases with increasing values of $W$. This patterns show that the higher the non-response rate, the greater the loss, this behaviors is practically justified.

It is indicated from Table 3.3, that: 
(i) for fixed values of $\left(W, f_{2}, \eta=0.25, \rho_{\mathrm{yx}}, \rho_{\mathrm{xz}}\right)$, the values of $\mu_{2}^{(0)}$ and $L_{21}$ increase as the value of $\rho_{\mathrm{yz}}$ increases. Thereby meaning is that if one use the information on a higher correlated auxiliary variable there is substantial gain in efficiency in the precision of estimators.

(ii) for fixed values of $\left(W, f_{2}, \eta=0.25, \rho_{\mathrm{yx}}, \rho_{\mathrm{yz}}\right)$, the value of $\mu_{2}^{(0)}$ and $L_{21}$ decrease with increasing values of $\rho_{\mathrm{xz}}$ increase.

(iii) for fixed values of $\left(W, f_{2}, \eta=0.25, \rho_{\mathrm{yz}}, \rho_{\mathrm{yx}}\right)$, the values of $\mu_{2}^{(0)}$ and $L_{22}$ increase as the value of $\rho_{y x}$ increases.

(iv) for fixed values of $\left(f_{2}, \eta=0.25, \rho_{\mathrm{yx}}, \rho_{\mathrm{yz}}, \rho_{\mathrm{xz}}\right)$, the values of $\mu_{2}^{(0)}$ decrease while the value of $L_{21}$ increases with increasing values of $W$. This indicates that with the larger non-response rate we need to select smaller sample size at the current occasion, which reduces the cost of the survey.

From Table 3.4 it is seen that:

(i) for fixed values of $\left(W, f_{2}, \eta=0.25, \rho_{\mathrm{yx}}, \rho_{\mathrm{xz}}\right)$, the values of $\mu_{2}^{(0)}$ increase while the value $L_{22}$ decreases with increasing value of $\rho_{\mathrm{yz}}$.

(ii) for fixed values of $\left(W, f_{2}, \eta=0.25, \rho_{\mathrm{yx}}, \rho_{\mathrm{zx}}\right)$, the values of $\mu_{2}^{(0)}$ decrease while the value of $L_{22}$ increase with increasing values of $\rho_{x z}$.

(iii) for fixed values of $\left(W, f_{2}, \eta=0.25, \rho_{\mathrm{yx}}, \rho_{\mathrm{yz}}\right)$ the value of $\mu_{2}^{(0)}$ and $L_{22}$ increase with increasing values of $\rho_{\mathrm{yx}}$.

(iv) for fixed values of $\left(f_{2}, \eta=0.25, \rho_{\mathrm{yx}}, \rho_{\mathrm{yz}}, \rho_{\mathrm{xz}}\right)$, the value of $\mu_{2}^{(0)}$ decrease while the value of $L_{22}$ increases with increasing values of $W$.

From Table 3.5 it is observed that:

(i) for fixed values of $\left(W, f_{2}, \eta=0.25, \rho_{\mathrm{yx}}, \rho_{\mathrm{xz}}\right)$, the value of $\mu_{1}^{(0)}$ increase while the value of $L_{1}$ decreases as the value of $\rho_{\mathrm{yz}}$ increases. This implies that if the variable under study and the auxiliary variable are more correlated, more negative loss (profit) is seen. 
(ii) for fixed values of $\left(W, f_{2}, \eta=0.25, \rho_{\mathrm{yx}}, \rho_{\mathrm{yz}}\right)$, the value of $\mu_{1}^{(0)}$ decrease while the values $L_{1}$ increases with increasing values of $\rho_{\mathrm{xz}}$.

(iii) for fixed values of $\left(W, f_{2}, \eta=0.25, \rho_{\mathrm{yz}}, \rho_{\mathrm{yx}}\right)$, the value of $\mu_{1}^{(0)}$ increase while the value of $L_{1}$ decreases as the value of $\rho_{\mathrm{yx}}$ increases.

(iv) for fixed values of $\left(f_{2}, \eta=0.25, \rho_{\mathrm{yx}}, \rho_{\mathrm{yz}}, \rho_{\mathrm{xz}}\right)$, the values of $\mu_{1}^{(0)}$ and $L_{1}$ decrease with increasing values of $W$.

It is shown from Table 3.6, that:

(i) for fixed values of $\left(W, f_{2}, \eta=0.25, \rho_{\mathrm{yx}}, \rho_{\mathrm{xz}}\right)$, the values of $\mu_{2}^{(0)}$ and $L_{2}$ decrease with increasing value of $\rho_{\mathrm{yz}}$.

(ii) for fixed values of $\left(W, f_{2}, \eta=0.25, \rho_{\mathrm{yx}}, \rho_{\mathrm{yz}}\right)$ the value of $\mu_{2}^{(0)}$ decrease while the value of $L_{2}$ increases with increasing value of $\rho_{\mathrm{yx}}$. Thus the higher the correlation between study variable over both occasions, the higher is the gain observed.

(iii) for fixed values of $\left(W, f_{2}, \eta=0.25, \rho_{\mathrm{yx}}, \rho_{\mathrm{zx}}\right)$, the value of $\mu_{2}^{(0)}$ increase while the value of $L_{2}$ decreases with increasing values of $\rho_{\mathrm{xz}}$.

(iv) for fixed values of $\left(f_{2}, \eta=0.25, \rho_{\mathrm{yx}}, \rho_{\mathrm{yz}}, \rho_{\mathrm{xz}}\right)$, the values of $\mu_{2}^{(0)}$ and $L_{2}$ decrease with increasing values of $W$.

\section{CONCLUSIONS}

It is observed from Table 3.1 to 3.6 that for all cases the percent relative loss in efficiency is observed when the non-response occurs at the current occasions. Tables 3.1 and 3.3 exhibit that the loss is observed due to the presence of non-response at the current occasion, but the formation of the estimators neutralizes the negative impact of non-response to the lager extent. It follows from Tables 3.2, 3.4, 3.5 and 3.6 that the proposed estimators $\left(P_{1}, P_{2}\right)$ yields higher amount of gain in efficiency over Hansen and Hurwitz (1946) estimator $\bar{y}_{n}^{*}$ as compared to the natural successive sampling estimator $T_{2}$ which substantiate the suitable use of power transformation over auxiliary variable. We have further observed from Tables 3.1 to3.6 that the performance of the proposed estimator $P_{2}$ is better than the estimator $P_{1}$. Thus our recommendation is in the favour of the suggested estimator $P_{2}$ for use in practice. 


\section{REFERENCES}

Biradar, R. S. and Singh, H. P. (2001): Successive sampling using auxiliary information on both the occasions. Calcutta. Statist. Assoc. Bull., 51, 243-251.

Chaudhary, R. K., Bathal, H.V. L. and Sud, U.C. (2004): On non-response in sampling on two occasions. Jour. Ind. Soc. Agril. Statist. , 58(3), 331-343.

Cochran, W. G. (1977): Sampling Technique 3rd Edition. Johan Wiley and Sons, New York.

Feng, S. and Zou, G. (1997): Sampling rotation method with auxiliary variable. Commun. Statist. Theo. Meth., 26(6), 1497-1509.

Hansen, M. H. and Hurwitz, W.N. (1946): The problem of non-response in sample surveys. Jour. Amer. Statist. Assoc., 41, 517-529.

Jessen, R. J. (1942): Statistical investigation of a sample survey for obtaining form facts. Iowa Agricultural Experiment Station, Road Bulletin, 304 Ames IA.

Kumar S., Singh, H. P., Bhougal S. and Gupta, R. (2011): A class of ratio-cum-product type estimators under double sampling in the presence of non response. Hacett. Jour. Math. Statist., 40(4), 589-599.

Patterson, H. D. (1950): Sampling on successive occasions with partial replacement of units. Jour Roy, Statist. Assoc., B (12), 241- 255.

Singh H.P., Sunil K. and Kazak M. (2010): Improved estimation of finite population mean using subsampling to deal with non response in two-phase sampling scheme. Commun. Statist. Theo. Meth., 39(5), 791-802.

Singh, G. and Priyanka, K. (2007): Effects of non-response on current occasion in search of good rotation patterns on successive occasions. Statistics in Transition, 8(2), 273-292.

Singh, H. P. and Kumar, S. (2008): A regression Approach to the estimation of the finite population mean in the presence of non-response. Aust. N. Z. Jour. Stat., 50(4), 1-14.

Singh, H. P. and Kumar, S. (2010): Estimation of population product in presence of non-response in successive sampling. Statistical Papers, 51(4), 975-996.

Singh, H. P. and Kumar, S. (2011): Effects of non-response on a class of estimators of population mean on current occasion in successive sampling on two occasions. Jour. Prob. Stat, Sci., 9(1), 69-89.

Singh, H. P. and Kumar, S. and Bhougal, S. (2011): Estimation of population mean in successive sampling by sub-sampling non-respondents. Jour. Mod., Appl. Statist. Meth., 10(1),51-60.

Singh, H. P. and Pal, S. K. (2015a): On the estimation of population mean in successive sampling. Int. Jour. Math. Sci. Appl., 5(1), 179-185.

Singh, H. P. and Pal, S. K. (2015b): On the estimation of population mean in rotation sampling. Jour. Statist. Appl. Pro. Lett., 2(2), 131-136.

Singh, H. P. and Pal, S. K. (2015c): On the estimation of population mean in current occasions in twooccasion rotation patterns. Jour. Statist. Appl. Prob., 4(2), 305-313.

Singh, H. P. and Pal, S. K. (2015d): Improved estimation of current population mean over two-occasions. Sri Lankan Jour. Appl. Statist., 16(1), 1-19. 
Singh, H. P. and Pal, S. K. (2016a): An efficient effective rotation pattern in successive sampling over two occasions. Commun. Statist. Theo. Meth., 47 (17), 5017-5027.

Singh, H. P. and Pal, S. K. (2016b): Search of good rotation patterns through exponential type regression estimator in successive sampling over two occasions. Italian Jour. Pure. Appl. Math. 36,567-582.

Singh, H. P. and Pal, S. K. (2016c): Use of several auxiliary variables in estimating the population mean in a two occasion successive sampling. Commun. Statist. Theo. Meth. 45(23), 6928-6942.

Singh, H.P. and Ruiz-Espejo, M. R. (2003): On linear regression and ratio-product estimation of finite population mean. The statistician, 52, 1, 59-67.

Singh, H. P. and Vishwakarma, G.K. (2007a): Modified exponential ratio product estimators for finite population mean in double sampling. Austrian Jour. Statist., 36 (3), 217 - 225.

Singh, H.P., and Vishwakarma, G.K. (2007b): A general class of estimators in successive sampling. Metron, 65(2), 201-227.

Singh, H.P., and Vishwakarma, G.K. (2009): A general procedure for estimating population mean in successive sampling. Commun. Statist. Theo. Meth., 38, 293-308.

Surya K. Pal

School of Studies in Statistics,

Vikram University, Ujjain-456010, M.P., India

Email: suryakantpal6676@gmail.com

Housila P. Sing

School of Studies in Statistics,

Vikram University, Ujjain-456010, M.P., India 